\title{
Secure Ad-Hoc Trust Initialization and Key Management in Wireless Body Area Networks
}

MING LI, Utah State University

SHUCHENG YU, University of Arkansas at Little Rock

JOSHUA. D. GUTTMAN, Worcester Polytechnic Institute

WENJING LOU, Virginia Tech

KUI REN, Illinois Institute of Technology

Body area network (BAN) is a key enabling technology in E-healthcare. An important security issue is to establish initial trust relationship among the BAN devices before they are actually deployed, and generate necessary shared secret keys to protect the subsequent wireless communications. Due to the ad hoc nature of the BAN and the extreme resource constraints of sensor devices, providing secure, efficient and userfriendly trust initialization is a challenging task. Traditional solutions for wireless sensor networks mostly depend on key pre-distribution, which is unsuitable for BAN in many ways. In this paper, we propose group device pairing (GDP), a user-aided multi-party authenticated key agreement protocol. Through GDP, a group of sensor devices that have no pre-shared secrets, establish initial trust by by generating various shared secret keys out of an unauthenticated channel. Devices authenticate themselves to each other under the aid of a human user who performs visual verifications. The GDP supports fast batch deployment, addition and revocation of sensor devices, does not rely on any additional hardware device, and is mostly based on symmetric key cryptography. We formally prove the security of the proposed protocols, and we implement GDP on a sensor network testbed and report performance evaluation results.

Categories and Subject Descriptors: C.2.0 [Computer-Communication Networks]: General—Security and Design; C.2.0 [Computer-Communication Networks]: Network Architecture and Design-Wireless communication, Network topology; C.4.0 [Computing Systems Organization]: Performance of Systems; K.4.6 [Operating Systems]: Security and Protection-Cryptographic controls; K.6.5 [Management of Computing and Information Systems]: Security and Protection

General Terms: Security, Design, Experiment, Body Area Networks, Efficiency

Additional Key Words and Phrases: Trust Establishment, Key Management, Usable Security, Device Pairing ACM Reference Format:

Li, M., Yu, S., Guttman, J. D., Lou, W., Ren, K. 2012. Secure Ad-Hoc Trust Initialization and Key Management in Wireless Body Area Networks. ACM Trans. Sensor Netw. 0, 0, Article 00 ( 0000), 35 pages.

DOI $=10.1145 / 0000000.0000000$ http://doi.acm.org/10.1145/0000000.0000000

This work was supported in part by the US National Science Foundation under grants CNS-0716306, CNS0831628, CNS-0746977, CNS-1117811 and CNS 0831963.

Authors' addresses: M. Li: Dept. of CS, Utah State University, 4205 Old Main Hill, Logan, UT 84322, email: ming.li@usu.edu; S. Yu: Dept. of CS, University of Arkansas at Little Rock, 2801 S. University Ave, Little Rock, AR 72204, email: sxyu1@ualr.edu; J. D. Guttman: Dept. of CS, Worcester Polytechnic Institute, 100 Institute Road, Worcester, MA 01609, email: guttman@wpi.edu; W. Lou: Dept. of CS, Virginia Tech, 7054 Haycock Road, Falls Church, VA, 24061, email: wjlou@vt.edu; K. Ren: Dept. of ECE, Illinois Institute of Technology, 3301 Dearborn St, Siegel Hall 319, Chicago, Illinois 60616, email: kren@iit.edu.

A preliminary version of this paper [Li et al. 2010] appeared in IEEE INFOCOM 2010 (The 29th Conference on Computer Communications).

Permission to make digital or hard copies of part or all of this work for personal or classroom use is granted without fee provided that copies are not made or distributed for profit or commercial advantage and that copies show this notice on the first page or initial screen of a display along with the full citation. Copyrights for components of this work owned by others than ACM must be honored. Abstracting with credit is permitted. To copy otherwise, to republish, to post on servers, to redistribute to lists, or to use any component of this work in other works requires prior specific permission and/or a fee. Permissions may be requested from Publications Dept., ACM, Inc., 2 Penn Plaza, Suite 701, New York, NY 10121-0701 USA, fax +1 (212) 869-0481, or permissions@acm.org.

(c) 0000 ACM 1550-4859/0000/-ART00 $\$ 10.00$

DOI 10.1145/0000000.0000000 http://doi.acm.org/10.1145/0000000.0000000 


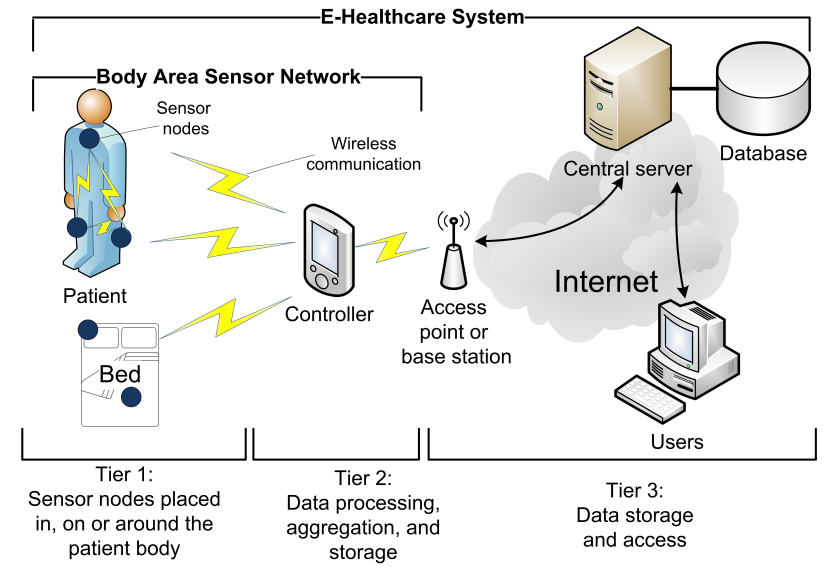

Fig. 1: A typical body area network and its relationship with the E-healthcare system. (c)2011, Ming Li)

(C)ACM, 2012. This is the author's version of the work. It is posted here by permission of ACM for your personal use. Not for redistribution.

\section{INTRODUCTION}

In recent years, the interoperable medical device (IMD) [Venkatasubramanian et al. 2010] has emerged as an enabling technique for modern E-healthcare systems, which would revolutionize hospital treatment [Lorincz et al. 2004; Hanson et al. 2009; Jovanov et al. 2005; Li et al. 2010a]. Traditional medical devices usually operate separately, while IMDs are able to interoperate with each other - they are small wearable or implantable medical devices that are capable of sensing, storing, processing and transmitting data via wireless communications. IMDs promise afford many advantages to the patient, including improved safety, more accurate diagnosis, and better context awareness for caregivers [Venkatasubramanian et al. 2010].

A network of IMDs is often referred to as a wireless body area network (BAN). It may consist of multiple IMDs of different types - they could be placed in, on or around a patient's body, while they fulfill the common goal of patient monitoring. In addition, a controller (a hand-held device like PDA or smart phone) is usually associated with each patient, which collects, processes, and transmits the sensor data to the upper tier of the network for healthcare records. A typical structure of the BAN and its relationship with the E-healthcare system is depicted in Fig. 1.

The BAN is designed to satisfy a wide range of applications, such as ubiquitous health monitoring (UHM) [Jovanov et al. 2005] and emergency medical services (EMS) [Lorincz et al. 2004]. The UHM features long-term and consistent monitoring of a patient's health status and surrounding environment, while the EMS requires realtime medical data collection and reporting.

Unlike conventional sensor networks, a BAN deals with medical information, which has stringent requirements for security and privacy. It is critical to protect this information from eavesdropping, malicious modification and unauthorized access, etc. Trust among the BAN devices is crucial for realizing these security requirements, including especially authenticated shared (symmetric) secret keys that enable cryptographic functions such as encryption and integrity check. However, in traditional wireless sensor networks (WSNs) the secret keys are usually pre-distributed before network deployment. The existing methods for key distribution in WSNs can be divided into 
several categories. (1) Rely on knowledge of the network topology [Perrig et al. 2002]; (2) Require less topology information but need the sensors to store a large number of keys [Eschenauer and Gligor 2002; Chan et al. 2003; Di Pietro et al. 2003; Du et al. 2005; Liu and Ning 2003; Liu et al. 2008]; (3) Assume the existence of root of trust from certain central entities [Zhu et al. 2003; 2006], or rely on public key infrastructure (PKI) [Malan et al. 2004].

However, key pre-distribution is not suitable for a BAN in several ways. First, the distribution chain of a medical sensor node may not be fully trusted by the end user: the devices could come out of the hands of different manufacturers and users. This rules out the first two types of pre-distribution methods in traditional WSNs, i.e., there will not exist shared keys or common security context within the IMDs before they arrive at end users. Second, a BAN is often formed in an ad hoc way with unpredictable topology, while "plug-n-play" is the ideal usability goal. It is hard for the users to distribute keys manually since they are usually not experts. Most existing works on user-aided key pre-distribution in WSNs involve cumbersome human efforts [Kuo et al. 2007; Law et al. 2010], and are not very user-friendly. Third, a central root of trust or a PKI would be impractical for BANs, not only because they require costly infrastructure, but also due to the high complexity involved in the revocation of nodes.

This gives rise to the problem of secure ad hoc initial trust establishment for a BAN, which happens before the BAN is actually deployed. Here we highlight several key differences between this and traditional key pre-distribution in WSNs. (1) Since secret keys are not assumed to be pre-distributed, trust must be established despite the lack of a common security context, and no central trusted parties as the root of trust except that the user trusts herself. In particular, in practice, a group of BAN devices must be correctly associated with the intended patient, lest the wrong medical data be collected. This requires the IMDs to be authenticated to each other and to the BAN controller, which forms the group securely. Secret keys which can belong only to the intended group should be generated. (2) The traditional authentication goal [Bellare and Rogaway 1994] only stipulates that each participant is assured that each message appears to come from the true identity that generated it. However, in a BAN since the wireless communication cannot be perceived by a human, in addition to traditional authentication it is desirable to let a human user physically make sure that the devices ultimately authenticated to each other include and only include the intended devices that s/he wants to participate, which is often referred to as demonstrative $i$ dentification [Chen et al. 2008; Lin et al. 2009] in usable security. To achieve this, the mechanism should be user-friendly, i.e., involving as few human interactions as possible. (3) BAN applications are usually time-critical which mandates the trust bootstrap process to be fast and scalable. For instance, in EMS an additional 5 minutes delay may result in a difference between life and death. Of course, overhead is an important concern since the medical sensor nodes are extremely resource-constrained.

A unique challenge is that, a secure communication channel shall be established out of an insecure channel for all the BAN devices upon their first meet, since IMD$\mathrm{s}$ communicate through wireless. This can be achieved by the so called secure device pairing concept that "pairs up" two devices [Li et al. 2010b]. A straightforward solution is to apply device pairing between the controller and each of the $N-1$ IMDs to establish individual keys, based on which the pairwise keys and group key can be derived. However, this requires about $N-1$ human interactions while each one needs tens of seconds. Many current device pairing techniques are designed for pairing only two devices, which will require many runs for a BAN. Many others are unsuited for IMDs with limited resources and little human interface. GAnGS [Chen et al. 2008] is an exception, but it still requires $N$ interactions. 
In this paper, we propose the group device pairing (GDP) protocol that establishes shared secret keys within a BAN out of nothing, i.e., it relies on neither prior shared secrets, nor common measurements, nor a PKI. GDP sets up an authenticated BAN group (including a shared group key and individual secret keys among devices), with much fewer human interactions (constant) than establishing authenticated individual shared keys between the nodes one at a time using traditional device pairing techniques. In GDP, each device authenticates itself to every other device in the group as a legitimate member, which can be verified visually by a human. With the initial shared secret keys, standard cryptographic methods can be applied to generate other secret keys on demand after BAN deployment.

\subsection{Our Contributions}

We propose a suite of novel schemes for secure ad hoc initial trust establishment and key management in BAN.

(1) We put forward GDP as the primary scheme for initial trust establishment that relies on zero prior security context. GDP is essentially a user-aided multi-party authenticated key agreement protocol, which combines the concept of device pairing and group key agreement in an unique way. We propose to use simultaneous comparison of synchronous LED blinking sequences on multiple resource constrained devices by human user as an auxiliary out-of-band (OOB) channel to authenticate the key exchange in the group. An authenticated group key and individual shared secret keys among IMDs can be set up for a batch of BAN devices only in one shot. As a secondary scheme, we also propose a pairwise device pairing (PDP) protocol which establishes a shared symmetric secret key between a controller and an IMD without relying on key pre-distribution. The GDP is particularly suitable for BAN, because it typically contains less than 100 IMDs and the devices are within one-hop range.

(2) GDP enables efficient key management after network deployment. Multiple types of keys can be derived on-demand based on the initial keys obtained during trust establishment before deployment. Also, dynamic operations, such as regular key updates, batch node addition and revocation are supported naturally by GDP. Our scheme is mostly based on symmetric key cryptography (SKC), thus having low communication and computation overhead.

(3) We formally prove the security of both schemes (GDP and PDP) based on the Bellare-Rogaway model [Bellare and Rogaway 1994], and give the security guarantees under the existence of a computational bounded adversary. The distinct features of our protocols and security proofs compared with other existing ones are: 1) Many previous protocols either require the use of non-malleable commitment schemes that involve heavy public key cryptography (PKC), or their security have not been formally proven. In contrast, our GDP and PDP both adopt commitment schemes that can be efficiently constructed from hash functions, while we prove their security without depending on the non-malleability of the commitments. 2) Our GDP protocol is also secure against compromised insider nodes, with the fewest communication rounds, while the only assumption underlying that is minimal, i.e., having a non-compromised controller.

(4) We carry out a thorough efficiency analysis for GDP, and implement it on a 10 node sensor network testbed to evaluate its performance. Experimental results show that initial trust establishment can be done within 30 seconds with low overhead in terms of time and energy consumption. GDP is secure yet practical. To the best of our knowledge, we are the first to propose, implement and test the feasibility of the 
visual OOB channel based on human comparison of simultaneous LED blinking patterns.

\subsection{Related Works}

The problem of secure initial trust establishment in BAN has received not much attention so far. In BAN, most previous works focus on security issues such as key management [Lorincz et al. 2004; Morchon et al. 2006; Malasri and Wang 2007], encryption [Lorincz et al. 2004; Malasri and Wang 2007; Tan et al. 2008], and access control [Tan et al. 2008]. However, it is a non-trivial issue to securely establish a secure communication channel among a BAN and associate it to the correct patient before any data communication happens.

1.2.1. Biometrical Methods. Biometrical values [Poon et al. 2006; Venkatasubramanian and Gupta 2010; Venkatasubramanian et al. 2010; Singh and Muthukkumarasamy 2007] have been used to establish a secure channel from which nodes can derive a common secret that associates the BSN to a specific patient's body. For example, electrocardiogram (EEG) and photoplethysmogram (PPG) has been exploited in [Poon et al. 2006; Venkatasubramanian and Gupta 2010; Venkatasubramanian et al. 2010]. This realizes initial trust establishment in a plug-and-play manner. However, it requires specific hardware for all the nodes to be equipped with the same sensing capability. Moreover, this biometrical channel is not always available since it does not apply to sensor devices that are not placed on the human body, for example, those that monitor the surrounding environment.

1.2.2. Key Generation based on Channel Characteristics. [Mathur et al. 2008] proposed to extract a secret key between two wireless devices out of an unauthenticated wireless channel using received signal strength indicator (RSSI). [Jana et al. 2009] evaluated the effectiveness of key extraction methods using RSSI in real environments. These methods do not rely on key pre-distribution, but the key generation rate is limited by the wireless channel and currently group key generation is not enabled.

1.2.3. Key Pre-distribution in BAN. Recently, the trust establishment in BAN is studied by [Keoh et al. 2009] under the context of secure sensor association. Each sensor node is associated with the controller one-by-one using public key based authentication, where a user compares LED blinking patterns to verify each association. However, their scheme assumes the existence of a trusted authority (TA), and still relies on the pre-distribution of public keys onto the sensor nodes. Also, it does not support batch deployment. In "message-in-a-bottle" [Kuo et al. 2007] and KALwEN [Law et al. 2010], a closed faraday-cage is employed as a secure channel, in which keying materials are pre-distributed to all the intended sensor nodes before deployment. Secure sensor association is achieved in the sense that the user is assured no attackers out of the cage can associate with the same patient. However, costly additional hardware is required and it is cumbersome to add new nodes.

1.2.4. Secure Device Pairing. Device pairing is a promising technique to generate a common secret between two devices that shared no prior-secrets with minimum or without additional hardware. It employs some low-bandwith out-of-band (OOB) channel to aid the authentication of information exchanged in the insecure wireless channel. Most proposed OOB channels rely on some form of human user participation. Wellknown examples include the "resurrecting duckling" [Stajano and Anderson 2000], "talking-to-strangers" [Balfanz et al. 2002], "seeing-is-believing" [McCune et al. 2005], Loud-and-clear [Goodrich et al. 2006] and short string comparison based key agreement schemes [Cagalj et al. 2006; Pasini and Vaudenay 2006]. In [Nithyanand et al. 2010; Kumar et al. 2009], the usability of device pairing protocols based on various 
OOB channels are evaluated. For a comprehensive survey, please refer to [Nguyen and Roscoe 2011].

1.2.5. Group Message Authentication Protocols. The idea of user-aided authentication has also been adopted in group message authentication protocols, where each group member wants to transfer an authenticated data copy from her device to each other's. For example, GAnGS [Chen et al. 2008] requires $O(N)$ human interactions, and also uses digital signatures, which increase computational complexity. In SPATE [Lin et al. 2009], this is done through comparing T-flags. Each group member carries out $N$ comparisons in parallel to authenticate other members' data. However, SPATE is specifically designed for message exchange and is not for group key agreement, and it lacks a formal security proof. [Laur and Pasini 2008] proposed a group message authentication and key agreement protocol (SAS-GAKA) based on comparison of short authentication strings (SAS). However, it does not achieve group demonstrative identification. Moreover, SAS and T-flags are not applicable for sensor nodes because they require richer device interfaces. Therefore, none of SPATE and SAS-GAKA is suitable for secure, fast, efficient and user-friendly initial trust establishment in BAN. In GDP, the whole group is authenticated and group key is generated in one shot (i.e., requires one-time visual comparison of synchronized LED blinking patterns).

The most recent work that is close to ours is GAP [Perkovic et al. 2011]. GAP is a user-aided group message authentication protocol that can be applied to wireless sensor networks. It also exploits the idea of synchronous LED blinking pattern as the OOB channel. The authors also discussed how to deal with semi-authenticated visual light channels which is orthogonal to our contribution. However, the security of GAP requires the use of non-malleable commitment schemes, where known constructions are much more inefficient than hash commitments used in this paper.

\section{PROBLEM DEFINITION}

\subsection{Network Model}

A BAN consists of a controller (gateway node) and a group of IMDs (medical sensor nodes). The size of the network varies, which may range from a few to the order of hundreds. Although the IMDs could be heterogenous in functionalities, we assume they are equipped with low-end, form-factor sensor nodes (e.g., comparable with Tmote). To meet the interoperability requirement, all of them are equipped with the same wireless communication interface, say ZigBee, and so does the controller. The sensors are limited in energy, communication, processing and storage capabilities, while the energy and computation resources of the controller are more ample.

The sensors may be placed in, on, or around the patient's body. Although there is no consensus on the communication technologies in BAN, the communication ranges in most current proposals are larger than $3 \mathrm{~m}$ (e.g. ZigBee). This is enough to assure that all nodes can be reached in one hop after deployment. Hence, we will assume a star topology. Each BAN has a patient, who may be regarded as its owner, as well as a user who sets up the network. The latter is often a nurse, but may be the patient herself.

\subsection{Design Requirements}

2.2.1. Security Goals. The initial trust establishment during pre-deployment should establish a group key and/or individual keys shared between each sensor and the controller, which can be used for the controller to securely broadcast messages to the BAN later, such as queries. For the design of the PDP and GDP (user-aided authenticated key agreement protocols), we have the following security goals: 
(1) Key secrecy and key confirmation [Ateniese et al. 2000]. For key secrecy, each group member should be assured that no non-member can obtain the group key. Key confirmation means each member is assured that the peers actually possess the same key.

(2) Group demonstrative identification. Suppose that a set $\mathcal{G}$ of devices is intended by the user to be the group associated with a specific patient. If a group formation process causes the set $\mathcal{G}^{\prime}$ of devices to derive the same group key, then the user should be able to physically verify that $\mathcal{G}$ and $\mathcal{G}^{\prime}$ are the same set.

Actually, this includes two properties - 1) key authenticity or consistency: each legitimate group member derives the same group key. If it also obtains individual shared keys, it must be assured that those keys come from the claimed true identities; 2) exclusiveness: the group includes only legitimate members and no attackers. This extends the "demonstrative identification" in [Balfanz et al. 2002; McCune et al. 2005], but is different from PAALP in GAnGS [Chen et al. 2008].

In addition, for the key management after deployment, it should have backward secrecy: a new group member should not learn about group keys in the past, and forward secrecy: a former group member should not discover subsequent group keys for existing members. The session keys may include pairwise keys shared between pairs of sensor nodes, so that they can securely distribute their data to other sensors. Sometimes, cluster keys are also needed in BAN.

\subsubsection{Usability Goals}

(1) Efficiency. A BAN is often consisted of low-end devices, relies on battery energy and is intended to last at least for several days [Hanson et al. 2009; Lorincz et al. 2004; Jovanov et al. 2005]. To match the low-capabilities of the sensors in BAN and to minimize energy consumption, it is important to minimize computation, communication and storage overhead. Therefore, expensive cryptographic functions such as public-key operations should be avoided whenever possible.

(2) Fast operation and user-friendliness. The initial trust establishment in a BAN should be fast, while involving as few and intuitive human interactions as possible. Especially, batch-deployment of devices should be supported.

(3) Error-proof. Since humans make mistakes, the procedure must be easy to follow. Also, the system should be able to detect errors or attackers and alert the user.

(4) Requires no additional hardware. In order to reduce the cost of the system, it is essential to use commercial-off-the-shelf(COTS) products, and to use fewer hardware components. For example, there should be no auxiliary devices. Also, the sensors usually do not have physical interfaces such as USB, because they may constrained form factors.

In addition, because the devices may be manufactured by different vendors which are hard to inter-operate, we assume there are no pre-loaded public keys, certificates, or pre-shared secrets among the devices in BAN. The sensors are used in a plug-andplay manner.

\subsection{Attack Model}

The attacker can either be an outsider or insider. An outsider does not compromise any devices in the intended BAN group, while an insider can compromise any of the sensor device. The attacker is able to eavesdrop, intercept, modify, replay or inject the wireless communication between any devices in range. The attacker can also compromise a certain number of sensor nodes after deployment.

The main goals of an attacker are: obtain the secret keys by eavesdropping; impersonate as a legitimate group member to join the group; prevent one or more legitimate 
group member to join the group; act as man-in-the-middle and try to split the intended group into two or more subgroups; maliciously modify the information contributed by legitimate group members so as to violate key authentication and disrupt the group. The attacker can also pose as multiple identities to join the group, which is a Sybil attack. We do not consider denial of service (DoS) attacks in this paper.

We assume only that the controller is not compromised during the initial trust establishment process (i.e., is trusted by the user) ${ }^{1}$. This is because the user can recognize his/her controller by password, and the controller is usually better kept and protected. Note that, devices do not trust each other before the initial trust establishment.

\section{BACKGROUND, NOTATIONS AND DEFINITIONS}

Table I. Frequently used notations

\begin{tabular}{l|l}
\hline$H()$ & A cryptographic hash function \\
$\mathcal{H}(m, r)$ & Digest function with input $m$ and key $r$ \\
$x \longleftarrow S$ & Choose $x$ uniformly from set $S$ \\
$E_{K}\{\cdot\}$ & Symmetric encryption with key $K$ \\
$\widehat{x}$ & The unauthenticated version of $x$ \\
$a \mid b$ & Concatenation of $a$ and $b$ \\
$M_{i}$ & The $i$-th group member \\
$\mathcal{G}$ & The group of devices intended to associate to a patient \\
$K_{G}, K_{i j}$ & The group key, the pairwise key between nodes $i$ and $j$ \\
$\mathcal{S}_{k}$ & A subgroup of index $k$ \\
$N$ & Total number of devices in the group \\
$\mathbb{Z}_{q}^{*}$ & Multiplicative group of prime order $q$ \\
$\mathbb{F}_{p}$ & Finite field of size $p$ \\
$n$ & The length of nonces \\
$\ell$ & Length of the short authentication string \\
\hline
\end{tabular}

\subsection{Communication Channels in Device Pairing}

In this paper, we consider secure device pairing protocols (or user-aided authentication protocols) with multiple communication channels. Usually there are two kinds of channels: one is the normal Dolev-Yao channel, the other is an auxiliary out-of-band (OOB) channel. In a Dolev-Yao channel, all the messages transmitted between two devices can be overheard, deleted, or modified by the adversary. Examples may include the wireless channel. In an OOB channel considered in this paper, messages cannot be modified or delayed from one to another session. The definition of the OOB channel corresponds to the "empirical channel" defined in [Nguyen and Roscoe 2011], and can be regarded as "authentic". The OOB channel is usually bandwidth-limited as compared with a Dolev-Yao channel. The former is represented as " $\leftrightarrow$ " in this paper, while the latter is denoted as “ $\Longleftrightarrow$ ".

Practical factors need to be considered when choosing the type of OOB channel in a device pairing protocol. In a BAN, sensor nodes may only have LED lights, beepers and buttons, but no interfaces such as camera, displays or keyboards; yet the controller may have all of them. Under this asymmetric setting, the methods in [McCune et al. 2005; Balfanz et al. 2002] are unable to achieve mutual authentication. Fortunately, the "Blink-Blink" (BB) pairing method proposed in [Prasad and Saxena 2008] was shown to be a practical approach. Briefly, both devices encode a short authentication string (SAS) obtained from a protocol run to a synchronized LED blinking pattern, where a ' 1 ' bit encodes to a "blink" (on) period and '0' bit encodes to an "off". Then the

\footnotetext{
${ }^{1}$ In the preliminary version of this paper [Li et al. 2010], we assumed all the devices to be benign during the pre-deployment phase; so the current assumption is much weaker.
} 
user compares the patterns and accepts the results if they are the same. The above is essentially a visual OOB channel between two devices, and we extend it to multiple devices in this paper.

\subsection{Commitment Schemes}

Commitment schemes are important cryptographic primitives that have been widely used in message authentication [Laur et al. 2005] and authenticated key agreement protocols [Cagalj et al. 2006; Pasini and Vaudenay 2006; Laur and Pasini 2008]. Typically, a commitment scheme consists of two algorithms: ${ }^{2}$

- Commit $(I N F O, x) \rightarrow(c, d)$, where $I N F O$ is public data, $x$ is $n$-bit private data, $c$ is the commitment value, and $d$ is an opening value. The algorithm is probabilistic.

- Open $(I N F O, c, d) \rightarrow x \in\{0,1\}^{n} \cup\{\perp\}$, which outputs the committed value $x$. If $c$ is not a valid commitment, then it returns $\perp$. This algorithm is deterministic, and correctness implies that for any $x \in\{0,1\}^{n}$, Open $($ INFO, Commit $(I N F O, x))=x$.

A commitment scheme should have two basic properties: hiding and binding. Their definitions are as follows.

DEFINITION $1\left(\left(\epsilon_{h}, T_{h}\right)\right.$-HIDING). Given $(c, I N F O)$, the probability that an adversary can correctly guess the value of $x$ before the opening value $d$ is revealed is upper bounded by $\epsilon_{h}$ in time $T_{h}$.

DEFINITION $2\left(\left(\epsilon_{b}, T_{b}\right)\right.$-BINDING). The probability that an adversary can open an commitment value $c$ to a different $x^{\prime}$ afterwards than the one committed by $c$ is upper bounded by $\epsilon_{b}$ in a time $T_{b}$.

In many existing user-aided authentication protocols [Perković et al. 2011; Laur and Pasini 2009; Laur and Nyberg 2006; Vaudenay 2005; Laur and Pasini 2008], the commitment schemes used are required to have a third property - non-malleability, which is stronger than the basic ones above. However, non-malleable commitment schemes are usually very inefficient in practice [Laur et al. 2005; Laur and Nyberg 2006], which will be unsuitable for low-end sensor nodes like Tmote. Fortunately, as we will show later, this property is not necessary for provable security of our proposed protocols. We instantiate the commitments using the following efficient construction from [Pass $2003]$ based on a cryptographic hash function ${ }^{3}$.

Definition 3 (HASh BASEd COMmitMent SCheme). Assume we have a cryptographic hash function $H$ that can be modeled as a random oracle: $\{0,1\}^{2 n} \rightarrow\{0,1\}^{l(n)}$, where $l(n) \leq$ poly $(n)$. Then we have the following scheme:

- Commit: given $x$, randomly picks $r \leftarrow\{0,1\}^{n}$, and compute $c=H(x, r)$.

- Open: let $d=(x, r)$. Output $x$ if $c=H(x, r)$.

The above scheme achieves hiding and binding [Pass 2003]. To commit to a longer message $x$, we can first hash it to $n$ bits using a collision-resistant hash function and then commit, which is a general method [Halevi and Micali 1996]. Therefore, with public data INFO and a message to be committed $(m)$, we can set $x=I N F O \mid m$ while

\footnotetext{
${ }^{2}$ In this paper we adopt the definition from [Nguyen and Roscoe 2011].

${ }^{3}$ In a few previous user-aided message authentication protocols, one-way hash functions (OHF) have been adopted as a practical alternative for commitment schemes [Zimmermann et al. 2006; Alliance 2006; Lin et al. 2009]. But to the best of the authors' knowledge, there have been no formal security proofs for such protocols up to date. In [Laur and Pasini 2009] a security proof was posed as an open problem. We here provide security proofs for our protocols.
} 
the hiding and binding properties defined in Defs. 1 and 2 still hold. We will denote the hash commitment using HCommit and HOpen.

\subsection{Digest Functions}

In this paper, we will make use of a digest function proposed by [Nguyen and Roscoe 2008 ; 2011]. The digest function is defined as a mapping:

Definition 4 (Digest FUnCTiON). $\mathcal{H}(m, k):\{0,1\}^{L} \times\{0,1\}^{n} \rightarrow\{0,1\}^{\ell}$ is a mapping where $m$ is the message to be digested and $k$ is the key. It shall have two properties:

(1) $\left(\epsilon_{u}\right.$-key-based uniformity) for any fixed $m$ and $y, \operatorname{Pr}_{k \in \in_{R}\{0,1\}^{n}}[\mathcal{H}(m, k)=y]=\epsilon_{u}$.

(2) ( $\epsilon_{r}$-no uniform compensation) for any fixed $\theta$ and $m \neq m^{\prime}, \operatorname{Pr}_{k \in R}\{0,1\}^{n}[\mathcal{H}(m, k)=$ $\left.\mathcal{H}\left(m^{\prime}, k \oplus \theta\right)\right]=\epsilon_{r}$.

The "key-based uniformity" says that, upon varying the key $k$, the output of the digest function should be uniformly distributed. And "no uniform compensation" means there should not exist $\theta$ such that it can always compensate the change in the digest output incurred by a different $m^{\prime}$ than $m$, for any varying key $k$.

A concrete construction is given in [Nguyen and Roscoe 2008] based on matrix product, where the ideal properties are achieved: $\epsilon_{u}=\epsilon_{r}=\frac{1}{2^{\ell}}$. Usually the output of a digest function is a short string, e.g., $\ell=16$ bits. Note that, it is similar to a universal hash function, but a universal hash usually concerns collision resistance w.r.t. the same key.

\subsection{Group Key Agreement Scheme}

A contributory group key agreement establishes a group key based on no pre-shared secret, where every member equally contributes one share of the group key. In this paper, we choose the unauthenticated group key agreement protocol (UDB) proposed by Dutta and Barua [Dutta and Barua 2008] as a primitive. It is based on DiffieHellman (DH) key agreement and is provably secure, and only requires 2 rounds of communication. However, its authenticated version uses digital signatures, which requires PKI and is unsuitable for BAN. We describe the UDB protocol for completeness in Fig. 2. $\mathbb{Z}_{q}^{*}$ is a multiplicative group of prime order $q$, where $g$ is a generator. Note that, $K_{G}=g^{x_{1} x_{2}+x_{2} x_{3}+\ldots+x_{n} x_{1}}$. Each node broadcasts two messages, and performs three modular exponentiations, $2 N-2$ modular multiplications and 1 modular division.

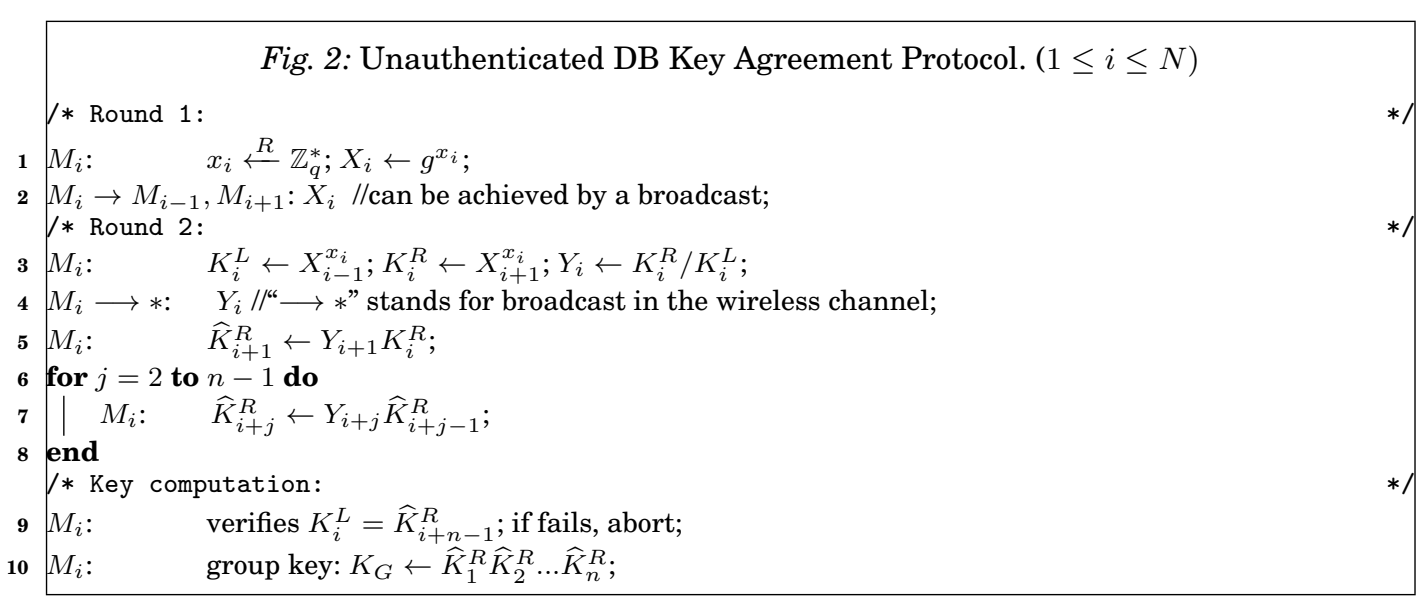




\section{SECURE AD HOC TRUST INITIALIZATION AND KEY MANAGEMENT FOR BAN}

\subsection{Overview}

Conceptually, the working cycle of a BAN mainly consists of three phases: predeployment, deployment and working phases. In the pre-deployment phase, the sensor nodes are bootstrapped for the first time after being purchased; thus, initial trust among sensors should be established in this phase. For this phase, we propose two schemes for securely establishing the initial shared secrets among a group of ad hoc BAN devices (including a controller and multiple sensors), without relying on any prior security context (or pre-shared secrets) among the devices. The core of the first scheme (Scheme-I) is a pairwise device pairing protocol (PDP), a.k.a. user-aided two-party authenticated key agreement, where a human user aids the authentication process by verifying simultaneous LED blinking patterns on both devices. By running the PDP protocol between the controller and each sensor one-by-one, each sensor derives an individual symmetric secret key with the controller. After that, the group key and pairwise keys can be established. The Scheme-I's complexity is $O(N)$ in terms of human effort. To improve upon it, we propose the group device pairing (GDP) protocol, a.k.a. user-aided multi-party authenticated key agreement. The GDP establishes authenticated group key and individual symmetric keys in a group of devices in one shot, with $O(1)$ human effort. Pairwise keys can also be subsequently obtained based on those keys. Both schemes are security enhanced versions of the corresponding ones in the preliminary version of this paper [Li et al. 2010]. In the GDP, the only additional assumption is that the controller is not compromised, which is reasonable since it is usually better protected by the human user. In the next section, we also prove the security of both PDP and GDP formally, while the GDP protocol is also secure against compromised sensor nodes inside the group.

In the deployment phase, nodes are actually deployed to designated places on/in/around the human body. Neighbor discovery is performed to form a BAN topology, pairwise keys are actually computed, and a logical key hierarchy is established. For the working phase, the regular functions (e.g. collecting and reporting medical data) are executed. We then discuss periodical key updates and how to handle node join/leave/revocation operations efficiently.

\subsection{Initial Trust Establishment via User-Aided Two-Party Authenticated Key Agreement}

In the pre-deployment phase, a group of sensor nodes and a controller picked by the user must be uniquely and securely associated to the patient they will serve for. This is done through establishing initial secret keys including individual keys and group key. Rather than pre-distributing key materials onto each device beforehand (where the whole process may not be fully trusted), our approach is based on the concept of device pairing, which does not rely on any prior security context among nodes. In this subsection, we first present a straightforward scheme (Scheme I) where the controller establishes a individual secret key with each sensor one-by-one via our PDP protocol.

4.2.1. The Pairwise Device Pairing Protocol. The PDP is depicted in Fig. 3. It is based on $\mathrm{DH}$ key agreement, and takes the $\mathrm{DH}$ public keys as part of the messages to be authenticated. The protocol essentially has three rounds; and the high-level idea can be described as "joint commitment before knowledge" [Nguyen and Roscoe 2011]: it means there is a point in every partial execution of the protocol such that both parties are committed to a value $D$ (in our case it is the SAS digest), but they do not yet know $D$; and in every successful completion of this partial execution, the parties are committed to the same value for $D$.

At first, $A$ and $B$ both generate a DH public value $\left(X_{A}\right.$ and $\left.X_{B}\right)$, and a random nonce $\left(r_{A}, r_{B}\right)$, respectively. In the first round, they compute hash commitments $\left(c_{A}, c_{B}\right)$ to 


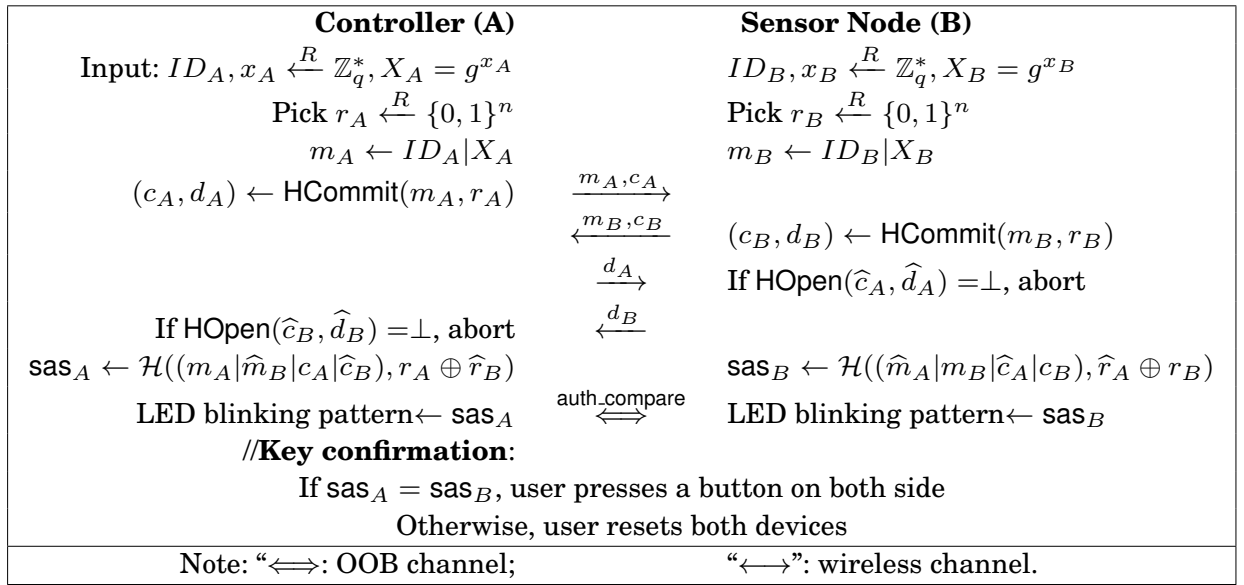

Fig. 3: User-aided two-party authenticated key agreement protocol (PDP) between the controller and a sensor node in Scheme I.

their corresponding nonces and IDs, and exchange the messages $m_{A}$ and $m_{B}$ along with the commitments. In the second round, the decommitment values are exchanged which reveal the nonces to each other. The above two rounds exchange messages using the wireless channel. In the third round, $A$ and $B$ both compute a SAS in order to authenticate $m_{B}$ and $m_{A}$, which is a digest based on their own and received messages and keys. The SASes are encoded into LED blinking patterns which are displayed synchronously over a visual OOB channel. The user compares the patterns (in an authenticated way), and accepts the authentication if they are the same. If authenticated, $K_{A B}=\widehat{X}_{B}^{x_{A}}=\widehat{X}_{A}^{x_{B}}=g^{x_{A} x_{B}}$. After that, the user needs to let both the controller and the sensor "know" the acceptance of the authentication result (key confirmation), by simply pressing a button on both devices.

There are some subtle points to be noticed. First, we have included the ID and DH public value of each party in its hash commitment. The ID is used to prevent the replay attack, where the adversary can copy a commitment of $A$ and later deliver it to $A$ again. And the inclusion of $\mathrm{DH}$ public value binds it with the commitment value, whose function will be more clear in the security proof. Second, we need to ensure a strict order of message exchange between the parties in order to synchronize both devices about the ending of phases. This can be done by announcing the devices' IDs before round I, and a node only sends its own data after receiving from the one with smaller ID. In the PDP, there is no constraint to the controller's ID. In contrast, we will see later in the GDP protocol, the controller's ID is required to be maximum. Third, in the SAS we have included both parties' IDs, DH public values and commitments, i.e., the protocol transcript. This also turns out to be an important factor for the security of both PDP and GDP protocols. Finally, the key confirmation can only be done manually, because otherwise there will be man-in-the-middle attacks at this stage. For example, in the preliminary version of this paper [Li et al. 2010], if the adversary establishes a different key with each of $A$ and $B$ before key confirmation, she will be able to deceive both $A$ and $B$ again at this stage.

4.2.2. Establishment of Group Key and Pairwise Keys. After $N-1$ individual shared keys are established, a group key $K_{G}$ is generated by the controller. To distribute the group key, the controller simply encrypts it $N-1$ times using the individual shared keys, and unicasts to each sensor node. Now, the user enters the ID of the patient into the 
controller, and associates the individual keys and the group key with this ID, which is also the ID of the BAN.

Next, in order to prepare for secure communication in the deployment phase and working phase, we need to distribute key materials to sensors so that they can establish pairwise keys afterwards. Here we use the Blundo's polynomial based key predistribution method [Blundo et al. 1993]. The controller first randomly generates a bivariate $t$-degree symmetric polynomial $f(x, y)=\sum_{i, j=0}^{t} a_{i, j} x^{i} y^{j}$ defined over a finite field $\mathbb{F}_{p}$ with $p$ being a large prime number ${ }^{4}$. The controller $C$ (the group member with largest ID, sometimes denoted as $M_{N}$ ) computes a univariate polynomial share for each node $M_{i}$ (with ID $i$ ): $f_{i}(y)=f(i, y)$. Then it encrypts and unicasts this to each sensor node:

$$
(m s g 1) C \longrightarrow M_{i}: i, E_{K_{N i}}\left\{f_{i}(y) \mid M A C_{K_{N i}}\left(f_{i}(y)\right)\right\} .
$$

where the message authentication code (MAC) provides authentication and integrity check, and $K_{C i}$ stands for the key shared between $C$ and $M_{i}$. Now, the pairwise key between $i$ and $j$ is: $K_{i j}=f_{i}(j)=f_{j}(i)=K_{j i}$.

In addition, in order for the controller to authenticate itself afterwards, the controller generates a one-way hash chain [Lamport 1981]: $\bar{k}_{n}, \bar{k}_{n-1}, \ldots, \bar{k}_{0}$, where $\bar{k}_{i}=$ $H\left(\bar{k}_{i+1}\right), 0 \leq i \leq n-1$. The controller distributes the commitment of the chain $\left(\bar{k}_{0}\right)$ to all sensor nodes:

$$
\text { (msg2) } C \longrightarrow M_{i}: E_{K_{G}}\left\{\bar{k}_{0} \mid M A C_{K_{G}}\left(\bar{k}_{0}\right)\right\} \text {. }
$$

\subsection{Initial Trust Establishment via User-Aided Multi-Party Authenticated Key Agreement}

In Scheme-I, associating sensor nodes one-by-one is very time-consuming, since each pair of LED blinking requires tens of seconds. Therefore, a more scalable and efficient method must be developed. The GDP below directly establishes initial secret keys in one shot, including a group key and individual keys among a group of devices through multi-party authenticated key agreement. The idea is to authenticate the messages exchanged in a group key agreement scheme with a human user's help, i.e., simultaneously comparing LED blinking patterns for a group of devices in an OOB visual channel.

We first propose the core protocol: GDP. We present it in two steps; firstly we give a multi-party message authentication protocol (MP-MAP), and then build the GDP based on the MP-MAP. The MP-MAP adopts similar design principles with the underlying MAP protocol of PDP, and their protocol structures resembles each other.

4.3.1. The Proposed MP-MAP. The MP-MAP for a group $\mathcal{G}$ is outlined in Fig. 4. It consists of four rounds. The first three rounds use wireless channel, while the fourth utilizes the visual channel.

Round 1 (wireless). In the counting \& group forming phase, the user $U$ would pick a group of $N$ devices and place them in close proximity. She chooses the controller device $M_{N}$ which has the largest ID among all devices (this can be ensured by assigning $I D_{N}$ a very large number), and enters the group member count $(N)$ into $M_{N}$ and indicates to start the protocol. Each member device $M_{i}$ broadcasts its own identity $I D_{i}$ to the group, and receives others' IDs. After a timeout, each $M_{i}$ sorts the pool of IDs in ascending order and keeps its own view of the group $\mathcal{G}_{i}$. In addition, the controller checks if the group size equals to $n$; if not, it will abort. The true group is denoted as $\mathcal{G}$, which can be perceived by the user.

Round 2 (wireless). In the commitment round, each $M_{i}$ generates a random nonce $r_{i}$ as its own share of digest key to generate the SAS in the end. Then $r_{i}$ is committed

${ }^{4}$ For example, we can use $p \approx 2^{80}$ to provide a 80 bit symmetric key. 


\begin{tabular}{|c|c|c|}
\hline & User designates controller $M_{N}$, which initiates the protocol & \multirow[b]{3}{*}{$\widehat{I D}_{i-1}, \widehat{m}_{i-1}, \widehat{c}_{i-1}$} \\
\hline $\begin{array}{r}\text { Round 1: } \\
\text { Counting\& } \\
\text { GroupForming } \\
\text { (wireless) }\end{array}$ & $\begin{array}{l}\text { Given } I D_{i} \text { (User enters group size } N \text { into } M_{N} \text { ) } \\
\text { Wait for }\left\{\widehat{I D_{j}}\right\}_{j \in \widehat{\mathcal{G}}} \text { until timeout } \\
\text { Set } \mathcal{G}_{i}=\left\{\widehat{I D_{1}}, \ldots, I D_{i}, \ldots, \widehat{I D_{N}}\right\} \text {, in ascending order of ID. } \\
M_{N} \text { will abort if }\left|\mathcal{G}_{N}\right| \neq N\end{array}$ & \\
\hline & \multirow[b]{2}{*}{$\begin{array}{l}\text { Pick } r_{i} \stackrel{R}{\leftarrow}\{0,1\}^{n} \\
\left(c_{i}, d_{i}\right) \leftarrow \text { HCommit }\left(I D_{i} \mid m_{i}, r_{i}\right) ; \text { record } \widehat{\mathbf{c}}_{i} \\
\text { Verify } \widehat{I D}_{j} \text { received after } \widehat{I D}_{j-1} \wedge \widehat{I D}_{j} \neq \widehat{I D}_{i}\end{array}$} & \\
\hline $\begin{array}{r}\text { Commitment } \\
\text { (wireless) }\end{array}$ & & \multirow{2}{*}{$\begin{array}{c}\stackrel{I D_{i}, m_{i}, c_{i}}{\widehat{I D}_{i+1}, \widehat{m}_{i+1}, \widehat{c}_{i+1}} \\
\ldots \\
\frac{\widehat{d}_{i-1}}{\longleftarrow \frac{d_{i}}{\widehat{d}_{i+1}}} \\
\ldots\end{array}$} \\
\hline $\begin{array}{r}\text { Round 3: } \\
\text { Decommitment } \\
\text { (wireless) }\end{array}$ & 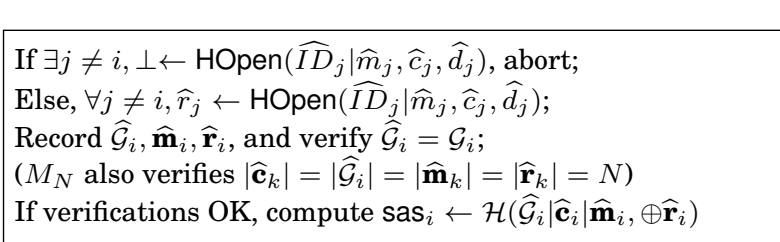 & \\
\hline $\begin{array}{r}\text { Round 4: } \\
\text { Auth. \& Confirm } \\
\text { (visual OOB) }\end{array}$ & $\begin{array}{l}\text { User verifies simultaneously LED: } \text { sas }_{i} \stackrel{?}{=} \text { sas }_{j}, \forall i \neq j \in \mathcal{G} \text {; } \\
\text { If verifications OK, user presses a button on every device. }\end{array}$ & sas $_{i}$ \\
\hline
\end{tabular}

Fig. 4: Multi-party message authentication protocol (MP-MAP) at each device $M_{i}$ : the message to be authenticated of each device is $m_{i}$.

along with the message $m_{i}$ and its ID, which are public data. Since the digest keys are hidden from the attacker in this round, all devices essentially have jointly committed to a SAS value that the attacker do not know. So the digest keys provide the randomness required for security. All devices send their commitments $c_{i}$ in order, i.e., $I D_{i-1}$ 's transmission must precede that of $I D_{i}$ 's, and each device can verify this order. The purpose is to provide device synchronization, i.e., they must agree on when one round ends. By using strict message ordering in rounds 2 and 3, the message sent by the device with largest ID serves as the synchronization signal. It prevents possible attacks that exploit the desynchronization, e.g., the one discovered in [Perkovic et al. 2011]. The controller will always be the last one to broadcast. Each device $M_{i}$ also keeps record of the set of received $\widehat{c}_{j} \mathrm{~s}-\widehat{\mathbf{c}}_{i}=\left\{\widehat{c}_{1}, \ldots, c_{i}, \ldots, \widehat{c}_{N_{i}}\right\}$, where $N_{i}$ should equal to $\left|\mathcal{G}_{i}\right|$.

Round 3 (wireless). In this round, each device $M_{i}$ reveals its committed digest key by broadcasting the decommitment value so that others can verify the validity of the commitment and obtain $\widehat{r}_{i}$ (they will check if $\widehat{I D}_{i}, \widehat{m}_{i}, \widehat{r}_{i}$ and $\widehat{c}_{i}$ are a valid messagecommitment pair). The controller, upon collecting all the other devices' commitments and digest keys, checks if the numbers of group members, commitments, messages and digest keys all equal to $N$ (the controller is assumed to be not compromised). In addition, each other device should check the consistency of the group IDs w.r.t. $\mathcal{G}_{i}$ collected at the beginning. After that, the SAS is computed at each $M_{i}$ as a digest of the protocol transcript, with the XOR of $M_{i}$ 's received set of $\widehat{\mathbf{r}}_{i}$ as digest key.

Round 4 (visual OOB). This round is when most of the human effort take place. Next, the SASes are encoded into synchronized LED blinking patterns for user comparison. The duration of the LED blinking depends on the number of bits of the SAS. 
User designates controller $M_{N}$, which initiates the protocol

Round 1: $\quad$ The same with round 1 in MP-MAP

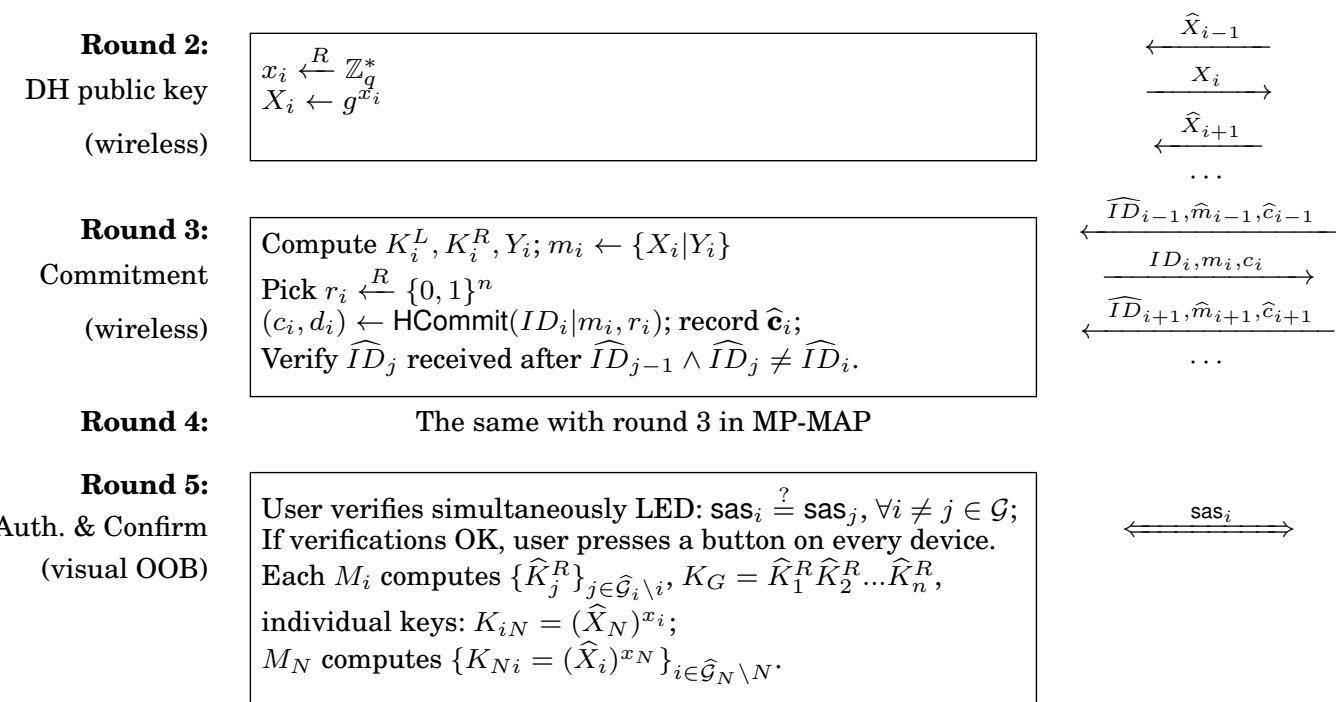

Fig. 5: The multi-party key agreement protocol (GDP) at each device $M_{i}$. It establishes a group key and sensors' individual keys with the controller.

Usually $16-20$ bits are enough for security. If all the patterns are the same, $U$ confirms that authentication succeeded by pressing a button on every device.

4.3.2. The Group Device Pairing Protocol. Next we describe the GDP protocol which combines the MP-MAP and the UDB group key agreement protocol. Round 1 is the same with that in MP-MAP. In round 2, a Diffie-Hellman (DH) public key $\left(X_{i}\right)$ is computed at each device, and is exchanged among all the devices in the group. In round 3, each device first computes its $Y_{i}$ value based on $X_{j}$ s received in round 2 , and then takes $X_{i} \mid Y_{i}$ as the message $m_{i}$ to be authenticated. Devices compute and exchange hash commitments in this round as in MP-MAP. Round 4 is the same as round 3 in the MP-MAP, which reveals the digest keys. Finally, in round 5, after confirming all the LED blinking patterns match, each device computes a group key based on all the previously received $X_{j}$ s and $Y_{j}$ s which should be already authenticated up to this point. In addition, as a byproduct, each sensor computes its individual key shared with the controller using the DH public key, and vice versa. As we will show in the next Section, the GDP achieves almost the same level of security as the PDP, with the same SAS length. Therefore, using the same amount of human effort as in the PDP, an authenticated group key and individual keys are all established.

4.3.3. Initial Trust Establishment via GDP. Now we describe some practical issues, e.g., how the GDP is applied to initial trust establishment in the BAN (or called secure sensor association). In reality, there is usually a limit to the number of LED blinking devices a human user can watch at the same time. We refer to this limit as $N_{\max }$. If the number of the intended group of devices for a BAN $N=|\mathcal{G}| \leq N_{\max }$, the user carries out one GDP for $\mathcal{G}$ to setup the group key $K_{G}$. If $N>N_{\max }$, the user randomly picks nodes from $\mathcal{G}$ in a batch to form smaller subgroups whose sizes are equal to $N_{\max }$ whenever possible. The GDP protocol is then executed for each subgroup $\mathcal{G}(k)$. The controller must be in every subgroup, so that it can establish a subgroup key $K_{G(k)}$ with each of 
them through GDP. When the last subgroup has only one sensor node left, Scheme I is automatically used to establish a pairwise key (however, it makes little difference to the user). After that the controller generates the final group key $K_{G}$ and broadcasts it using encryption to each subgroup: $E_{K_{G(k)}}\left\{K_{G}|\mathcal{G}| M A C_{K_{G(k)}}\left(K_{G} \mid \mathcal{G}\right)\right\}$, where $\mathcal{G}=\cup_{k} \mathcal{G}(k)$ and $|\mathcal{G}|=N$.

After the sensor association is successfully done, the group of devices need to setup the pairwise keys among them. There are two options. The simplest way is to reuse the DH public keys and let each $M_{i}, i \in \mathcal{G}$ compute $K_{i j}=\left(X_{j}\right)^{x_{i}}, \forall j \in \mathcal{G} \backslash i$. But this incurs additionally $N-2$ exponentiation operations for each sensor device (except the individual key computation), which is not desirable for resource-constrained sensors. The other way is to use the method in Sec. 4.2.2, i.e., let the controller broadcast material to each sensor which is encrypted under the sensor's individual key. And then each sensor computes the shared pairwise keys with others on its own. In this way, exponentiation operations are replaced with less costly field multiplication operations.

\subsection{Deployment and Thereafter}

The deployment phase establishes the pairwise and logical keys. Upon deployment, each node $M_{i}$ first performs neighbor discovery. For each neighbor $M_{j}, M_{i}$ computes the pairwise key $K_{i j}$ as previously mentioned. In practice, in order to save storage space, a node can merely store the pairwise keys that it uses frequently, while computing the other pairwise keys on-demand.

Then, the logical keys are derived naturally from the subgroup keys in GDP, which are used to form a logical key hierarchy (LKH). The LKH [Wong et al. 1998] has been proposed to achieve efficient key revocation. Since the LKH is a balanced binary tree, the message overhead for key revocation is $O\left(\log _{2}(N)\right)$. However, it is not very efficient for batch node addition or removal.

To avoid this drawback, we use a constant depth $(d=3)$, variable branch and balanced key tree (Fig. 6). Each internal node stands for a logical key, and each leaf node corresponds to the individual key of a sensor node. So we have $k_{0,0}=K_{G}$ and $k_{2, i}=K_{C, i+1}$. The keys $k_{1, i}=K_{G(k)}$ which are the subgroup keys derived in the end of GDP. The branch of the root $\mu_{0,0}$ equals to the number of subgroups, while the branch of a second level node $\mu_{1, i}=|\mathcal{G}(i)|$. The controller $C$ has the information of the entire key tree. Note that, no messages are needed to transmit the logical keys for the tree in our scheme.

Note that, our scheme can be easily extended to BANs with cluster topologies, since we can predict which nodes will form a cluster and thereby a subgroup by looking at their functionalities. For example, the use of several sensor nodes connected to 30 motion sensors are reported in [Van Laerhoven et al. 2002] to detect patient's acceleration and gait. A simple clustered BAN topology is shown in Fig. 7. Some nodes form clusters (e.g., $M_{4}, M_{5}$ and $M_{6}, M_{7}, M_{8}$ ), while others are independent with each other $\left(M_{1}, M_{2}, M_{3}\right)$. In order to save energy, the controller directly communicates with cluster heads and non-clustered nodes. In this case, the cluster keys will be the logical keys and the subgroup keys at the same time. We can use GDP to setup the cluster key for both clusters, and use PDP to establish individual keys for each non-clustered node.

After that, the BAN is ready to function. In summary, now a sensor node $M_{i}$ has the following key (material)s: $K_{G}, K_{i, N}, K_{G(k)}, f_{i}(y), \bar{k}_{0}$. Since the keys may be compromised by cryptanalysis afterwards, we need to introduce sessions for the working phase - time periods across which keys are updated regularly. The above keys are all treated as keys in session 0. A key $K$ in session $i$ is denoted as $K(i)$.

4.4.1. Session Key Update. Periodically, the controller broadcasts a update message to the network. It is authenticated using the local broadcast authentication method [Zhu 


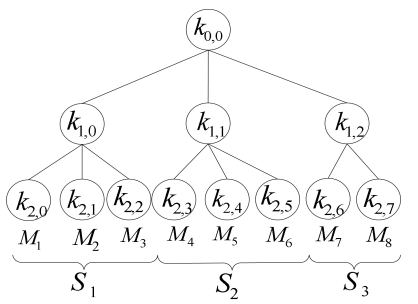

Fig. 6: A logical key tree for a BAN of 9 nodes $\left(N_{\max }=3\right)$. A key is indexed by its level $\lambda$ and branch number $\mu . \mathcal{G}(k)$ refers to a subgroup.

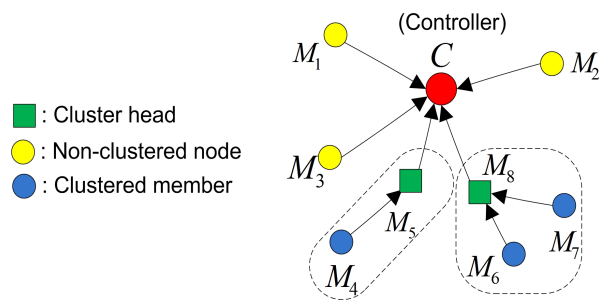

Fig. 7: A simple clustered BAN topology.

et al. 2003], since we assume the BAN is one-hop. The controller first updates $f(x, y)$ : $f_{i+1}(x, y)=f_{i}(x, y)+\Delta_{i+1}$, where $\Delta_{i+1} \stackrel{R}{\leftarrow} \mathbb{F}_{p}$. Then, it updates the logical keys as $k_{0,0}(i+1)=H\left(k_{0,0}(i)\right), k_{1, \mu}(i+1)=H\left(k_{1, \mu}(i)\right)$, and broadcasts the following:

$$
\begin{array}{r}
m s g 3 \leftarrow \text { "Update to session } i+1 " \mid \Delta_{i+1} \\
C \longrightarrow *: E_{k_{0,0}(i)}\{m s g 3\}, \bar{k}_{i+1}, M_{\bar{k}_{i+1}}(m s g 3) .
\end{array}
$$

Then, each sensor can authenticate $C$ by verifying that $H\left(\bar{k}_{i+1}\right)=\bar{k}_{i}$.

Next, all sensor nodes update all the keys in its memory as the controller does. For the pairwise keys, node $u$ computes $f_{u, i+1}(y)=f_{u, i}(y)+\Delta_{i+1}$. This achieves the update of all $\frac{N(N-1)}{2}$ pairwise keys through only one broadcast message.

\subsection{Membership Management}

4.5.1. Node Join. Adding one node is easy; we can just perform one device pairing using Scheme I. We will elaborate on how GDP supports efficient batch node addition.

Step 1 . Before $l>1$ new nodes join the BAN during session $i$, they are reset by the user (all dynamic memories are lost) and assumed to be benign.

Step 2. Before they are deployed, the same steps in GDP are performed by treating them as a new group, where the controller obtains the temporary group key $K_{G}^{T}$ and all the logical keys.

Step 3. The controller advances the existing BAN to session $i+1$ without waiting until the end of session $i$. To this end, all nodes do the same thing as in session key update.

Step 4. The controller pre-distributes new polynomial shares $f_{v, i+1}(y)$ for each new node $v$. Also, it encrypts $K_{G}(i+1)$ and $\bar{k}_{i+1}$ using $K_{G}^{T}$ and broadcasts to the new nodes. A new key tree can then be derived that includes the new nodes. Then, the new nodes are deployed.

4.5.2. Node Leave/Revocation. Upon single node leave or revocation during session $i$, the group key, logical keys and pairwise keys are renewed to exclude the leaving node. 
The controller randomly generates a new group key $K_{G}(i+1)$. All the logical keys on the tree path of the leaving node are refreshed. For example, in Fig. 6 , say $M_{1}$ is revoked. Then, the controller sends the following messages:

$$
\begin{array}{r}
C \rightarrow M_{2}: E_{k_{2,2}}\left\{k_{1,0}(i+1)\right\} ; \\
C \rightarrow M_{3}: E_{k_{2,3}}\left\{k_{1,0}(i+1)\right\} ; \\
C \rightarrow M_{2}, M_{3}: E_{k_{1,0}(i+1)}\left\{k_{0,0}(i+1)\right\} ; \\
C \rightarrow M_{4}, M_{5}, M_{6}: E_{k_{1,1}(i+1)}\left\{k_{0,0}(i+1)\right\} ; \\
C \rightarrow M_{7}, M_{8}: E_{k_{1,2}(i+1)}\left\{k_{0,0}(i+1)\right\} ;
\end{array}
$$

where $k_{1,1}(i+1)=H\left(k_{1,1}(i)\right), k_{1,2}(i+1)=H\left(k_{1,2}(i)\right)$. After that, the controller sends the updated polynomial share $\left(\Delta_{i+1}\right)$ to all nodes using authenticated broadcast. Thus, the revoked node cannot obtain the new group key and the updated polynomial share. It is straightforward to see how the above is done when batch node leave event happens, for which we will analyze the efficiency in Sec. 6 .

\section{SECURITY ANALYSIS}

For the authenticated key agreement (AKA) protocols in this paper, there are essentially two security goals: key secrecy and key authenticity. A basic secrecy goal is defined w.r.t. a passive adversary, i.e., an eavesdropper should have negligible advantage in deriving the shared key $K_{A B}$. In PDP, the only information sent over the wireless channel for the derivation of $K_{A B}$ is the set of the $X_{i} \mathrm{~s}$. Thus, key secrecy with a passive adversary amounts to that of Diffie-Hellman key exchange, which follows from the assumption that the Decisional Diffie-Hellman (DDH) problem is intractable. In the GDP protocol, a similar passive secrecy guarantee follows from the secrecy of the UDB key agreement protocol [Dutta and Barua 2008].

Thus, key authenticity will be the AKA protocol security goal we study in the remainder of this section. The cores of our AKA protocols are their corresponding message authentication protocols (MAPs). In the following, we focus on defining and proving the security of MAPs. The security of an AKA protocol follows from the security of it$\mathrm{s}$ underlying MAP and the security of the key agreement protocol against a passive adversary. ${ }^{5}$

Without loss of generality, we state the security definition of MAP using the multi-party scenario. Assume the group consists of $N$ parties (devices): $\mathcal{G}=$ $\left\{I D_{1}, I D_{2}, \ldots, I D_{N}\right\}$; for simplicity we use $i$ to represent $I D_{i}$. Each party $i \in \mathcal{G}$ has some message $m_{i}$ to be authenticated to all the rest parties in $\mathcal{G}$, for example in the PDP $m_{i}=\left\{I D_{i}, X_{i}\right\}$, while in GDP $m_{i}=\left\{I D_{i}, X_{i}, Y_{i}\right\}$.

Next, we define secure message authentication of a MAP based on the notion of "matching conversations" introduced by [Bellare and Rogaway 1994], where the detail$\mathrm{s}$ are provided in Appendix A. The following security definition captures the intuition that if a MAP is secure, then the only way that an adversary can make all parties accept at the end of a protocol run is to faithfully relay all the messages. We will use $\widehat{\mathbf{m}}_{i}$ to denote $i$ 's received vector (ordered set) of messages $\left\{\widehat{m}_{1 i}, \ldots, \widehat{m}_{i-1 i}, m_{i}, \widehat{m}_{i+1 i}, \ldots, \widehat{m}_{N i}\right\}$, and similarly $\widehat{\mathbf{c}}_{i}$ stands for the vector of received commitments by $i$, etc.

\footnotetext{
${ }^{5}$ To show this, the modular approach proposed by Bellare et al. [Bellare et al. 1998] can be applied. Specifically, It assumes two adversary models - the authenticated link model (AM) and un-authenticated link model (UM). If a protocol is proven to be secure in the AM, then it can be shown to be secure in the UM provided that each message transferred between the parties is authenticated by a protocol called message transfer (MT) authenticator. In our setting, by saying "security of the key agreement protocol" we mean its unauthenticated version (e.g., original Diffie-Hellman) should be secure in the AM, while the MAP can be regarded as an MT-authenticator.
} 
Definition 5 (Secure Message Authentication). We say that $\Pi$ is a $(\epsilon, T)$ secure message authentication protocol with a group of participants $\mathcal{G}(|\mathcal{G}| \geq 2)$, if for any $T$-time adversary $\mathcal{A}$,

(1) (Matching conversations $\Rightarrow$ acceptance) If all pairs of parties in $\mathcal{G}$ have jointly matching conversations, then all parties accept.

(2) (Acceptance $\Rightarrow$ matching conversations) Letting $\operatorname{Adv}_{\Pi}(\mathcal{A})=\operatorname{Pr}[$ All-accept $\wedge$ No-Matching $\left.{ }^{\mathcal{A}}\right]$, where No-Matching ${ }^{\mathcal{A}}$ refers to the event that the conversations are not jointly matching, we have $\operatorname{Ad} v_{\Pi}(\mathcal{A}) \leq \epsilon$.

In (2), we may use the uncorrupted group $\mathcal{N}=\mathcal{G}$, in which case we speak of the adversary as an outsider. Alternatively, we may choose $\mathcal{N} \subsetneq \mathcal{G}$, and speak of an insider adversary. In a two-party MAP, one does not need to consider one of the parties being compromised, because then there is nothing to prove. Thus we only discuss node compromise for the multi-party protocols.

\subsection{Security of the PDP}

We will refer to the message authentication protocol underlying the PDP as "two-party MAP (TP-MAP)". We first state the following theorem.

THEOREM 1. Assume that the digest function satisfies $\epsilon_{u}$-key-based uniformity and $\epsilon_{r}$-no uniform compensation. If the hash commitment scheme is $\left(\epsilon_{h}, T_{h}\right)$-hiding and $\left(\epsilon_{b}, T_{b}\right)$-binding, the TP-MAP is $\left(\max \left\{\epsilon_{u}, \epsilon_{r}\right\}+\epsilon_{h}+2 \epsilon_{b}, 2 T_{b}+T_{h}+O(1)\right)$-secure.

Proof. Please refer to Appendix. B.

Security interpretation. The security levels achieved by the TP-MAP (and the MPMAP as we will see) depend mainly on the SAS's length $\ell$. This is because the adversary's deception probability is dominated by either $\epsilon_{u}$ or $\epsilon_{r}$, which should equal to $2^{-\ell}$ given an ideal digest function. While $\epsilon_{h}, \epsilon_{b}$ reflect the security of hash commitment, which use long nonces. Their values are approximately $2^{-n}$, orders smaller than $2^{-\ell}$.

\subsection{Security of the GDP}

The MP-MAP can be proven as secure as the TP-MAP under the Bellare-Rogaway model, even when there exist compromised devices (insider attack). Our assumption is that the controller is not compromised, but any other sensor could be compromised by the adversary ${ }^{6}$.

THEOREM 2. Assume that the digest function satisfies $\epsilon_{u}$-key-based uniformity and $\epsilon_{r}$-no uniform compensation. If the hash commitment scheme is $\left(\epsilon_{h}, T_{h}\right)$-hiding and $\left(\epsilon_{b}, T_{b}\right)$-binding, the MP-MAP is $\left(\max \left\{\epsilon_{u}, \epsilon_{r}\right\}+\epsilon_{h}+2 \epsilon_{b}, 2 T_{b}+T_{h}\right)$-secure.

Proof. Please refer to Appendix. C.

Remarks. The MP-MAP and TP-MAP's security proofs are similar, and they both belong to the directly binding category [Nguyen and Roscoe 2011]. Interestingly, we can summarize several principles underlying both multi-party and two-party version of the MAP protocol in this paper. They are: (1) they both follow the joint-commitment before knowledge principle, where the hash commitment only needs two properties - hiding and binding; (2) they both have the strict order of message exchanges in each round; (3) they both use a digest function with the key-based uniformity and no uniform compensation properties defined in Section. 3.3; (4) they have both bound the message $m_{i}$ to the commitment, and digest for SAS involves all protocol transcript.

${ }^{6}$ For a MP-MAP to make sense, there must be at least two non-compromised devices. 


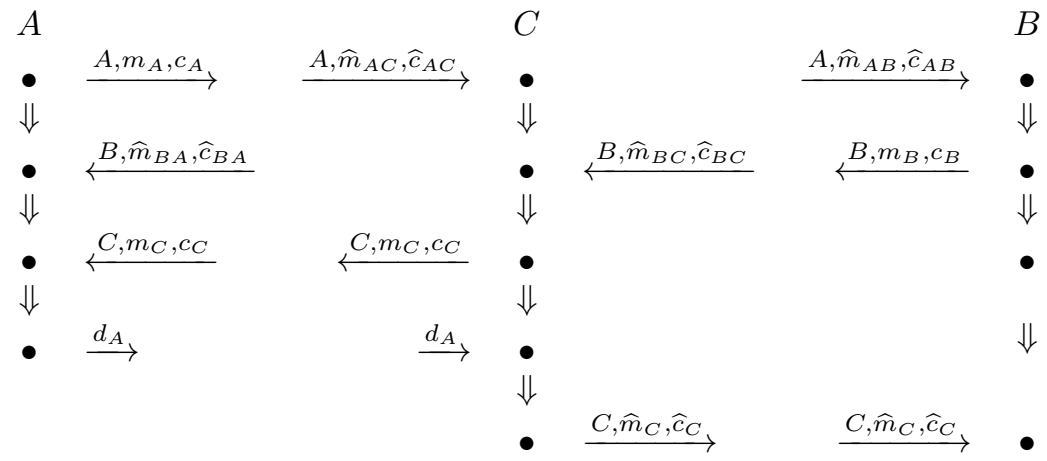

Fig. 8: A potential attack scenario against three party MAP, if the controller $(C)$ is compromised.

5.2.1. Security Intuition of the Role of Member Count. The member count information plays an important role in achieving exclusiveness (or demonstrative identification), i.e., the group authenticated in the end includes only the devices the user sees in front of her, which excludes any outsider attacker. If there is no member count information, exclusiveness cannot be achieved, as is the case in [Laur and Pasini 2008]. This is because before the group of devices meets with each other, they do not know the member list in advance. An attacker $\mathcal{A}$ can thus claim it is one of the group members and inject her DH public key share, trying to obtain the group key. Then the actual group becomes $\widehat{\mathcal{G}}=\mathcal{G} \cup \mathcal{A}$, while for members in $\mathcal{G}$, they still have the same SAS values. While the only sign that the user perceives is the LED blinking patterns on the sensor nodes, she will accept $\widehat{\mathcal{G}}$ as authenticate. However, with the count information, this attack can be defeated. First, if $N+1$ key shares are received by the controller, GDP will abort, assuming that the user counts correctly. Second, if $M_{N}$ only receives $N X_{i}$ s and $Y_{i}$ s from $\mathcal{G}$, but $\mathcal{G} \backslash M_{N}$ all receive $N+1$ key shares from $\mathcal{G} \cup \mathcal{A}, \mathcal{A}$ will not be able to derive the same key with all $j \in \mathcal{G}$, thus have no gain. Even if $\mathcal{A}$ carries out such attack to disrupt the group, it will not be able to make all the SASes equal due to the properties of the digest function.

5.2.2. Security Intuition Against Compromised Devices. Here we provide more insight into why GDP is secure against compromised devices. We illustrate it using a potential attack in reminiscence of the one discovered in [Perkovic et al. 2011], if the controller (device with largest ID) is compromised.

Suppose there are three devices: $A, B$ and $C$. Controller $C$ is under the full control of the adversary, i.e., it can launch active attacks in the wireless channel. Depicted in Fig. 8, $C$ tries to impersonate $B$ to $A$ and vice versa, but it does not try to break the group exclusiveness. $C$ 's goal is to make all the SASes equal. In the first move, after seeing $c_{A}, C$ constructs new commitments $\widehat{c}_{A C}$ and $\widehat{c}_{A B}$ with $\widehat{r}_{A C}, \widehat{r}_{A B}$ known by itself. Then after $B$ sends $c_{B}, C$ does the similar thing to the above. In the third move, $C$ sends $C, m_{C}, c_{C}$ only to $A$ to trick $A$ send its decommitment $d_{A}$, so that $C$ will know $r_{A}$ before this round ends. At this point, $C$ knows all the random nonces received/generated at $A$, and also all the received/generated data at $A$, which leads to the revealing of sas $_{A}$ in advance. What remains for $C$ is to compute $\widehat{c}_{C}$ and $\theta$ offline (after seeing $c_{B}$ ), such that $\widehat{c}_{C}$ opens to an $\widehat{r}_{C}=r_{B} \oplus \theta$ where $r_{B}$ is not known by $C$, which makes sas ${ }_{B}=$ sas $_{A}$. Assuming this can be done (since our hash commitment does not preclude malleability), $C$ can make all SASes equal while deceiving both $A$ and $B$. 
In the above attack, the attacker knows the last digest key $r_{N}$. However, if the controller is not compromised but the attacker compromises any other device with smaller ID (e.g. $B$ ), there is no way for she to obtain the value of $r_{N}$ before the commitment round ends (except by breaking the hiding property with negligible probability). So there is no way to know the SAS of the controller beforehand, which also means she cannot compute the SAS of other devices (e.g., A) offline to make SASes equal.

Therefore, the key factor for MP-MAP to be immune from insider attacks is that, the uncompromised controller is mandated to be the device with largest ID. Note that, in [Perkovic et al. 2011] the similar problem is dealt with by adding another round between the commitment and decommitment rounds. Our scheme keeps the number of rounds to the minimum.

\subsection{Security of Key Management}

5.3.1. Secrecy of the Key Polynomial. This is ensured to be unconditionally secure and resists up to $t$ colluding attackers [Blundo et al. 1993]. If more than $t$ polynomial shares are collected, $f(x, y)$ can be reconstructed using bivariate Lagrange interpolation. Therefore, we set $t$ as the maximum number of nodes in the BAN. For example, $t=50$ is usually enough. In this case, even if all the sensors are compromised, $f(x, y)$ is secure and we can replace compromised nodes by new ones, as long as the total number of nodes is smaller than $t$.

5.3.2. Backward Secrecy. For a new group member $v$ joined during the $i$-th session, the new group key sent out by the controller is $K_{G}(i+1)$. It is infeasible for $v$ to derive $K_{G}(i)$, since it requires to break the pre-image resistance property of hash function.

5.3.3. Forward Secrecy. For a revoked former group member $v$, since the new group key $K_{G}(i+1)$ is randomly generated by the controller and is securely delivered to the remaining group members, $v$ can only randomly guess the value of $K_{G}(i+1)$.

5.3.4. Key Update and Revocation. A revoked group member must not be able to communicate with existing members. Because the value $\Delta(i+1)$ is randomly chosen from $F_{p}$ and is encrypted thus is not known to revoked members, $v$ can only guess it randomly. The success probability is $1 / p$. For $v$, without knowing $\Delta(i+1)$, even if it possesses $f_{v, i}(y)$, it cannot derive $f_{v, i+1}(y)$, therefore cannot obtain pairwise keys with any legitimate node.

\section{EVALUATION}

In this section, we analyze the efficiency of our device pairing and key management protocols. We first compare the overheads with an existing scheme, and then report our implementation of GDP and experimental results.

\subsection{Computation and Communication Efficiency of GDP}

It is important for the trust establishment in a BAN to have both low computation and communication costs. A common reason is to keep low energy consumption for resource-constrained sensor devices. But more importantly, performing complex computations would increase the protocol run time dramatically, which is not tolerable for medical monitoring applications - especially under emergency situations. Many existing group message authentication (GMA) protocols [Vaudenay 2005; Laur and Nyberg 2006; Laur and Pasini 2008; 2009; Perković et al. 2011] require the adoption of a "non-malleable" commitment scheme, which is usually constructed based on number- 


\begin{tabular}{|c|c|c|c|}
\hline & Decomposition & SAS-GMA (bits) & MP-MAP (bits) \\
\hline & $I D$ & $N \cdot|I D|$ & $2 N \cdot|I D|$ \\
Commu. cost & commit & $N \cdot c_{1} \cdot q$ & $N \cdot n$ \\
& decom. & $N \cdot\left(c_{2} \cdot q+n+|I D|\right)$ & $2 N \cdot n$ \\
& message & $N \cdot|m|$ & $N \cdot|m|$ \\
\hline \multirow{3}{*}{ Comput. cost } & hash $H(\cdot)$ & $N^{2} \cdot n \cdot(|I D|+|m|)$ & $N^{2} \cdot n \cdot(|I D|+|m|+n)$ \\
& commit/decom. & $N \cdot\left(c_{1}^{\prime}+c_{2}^{\prime}\right) \cdot$ mod_exp & $N \cdot n \cdot(|I D|+|m|+n)$ \\
& sas & universal hash & digest function \\
\hline
\end{tabular}

Table II: Comparison of MP-MAP and SAS-GMA in terms of overall communication and computation. $N$ : number of devices; $q$ : length of group element in a non-malleable commitment scheme.

theoretic assumptions and incurs intensive computation ${ }^{7}$ [MacKenzie and Yang 2004; Vaudenay 2005; Laur and Nyberg 2006]. A representative scheme of this kind is the SAS-GMA protocol proposed in [Laur and Pasini 2008; 2009], which we will compare with. In terms of computation, the biggest advantage of our MP-MAP is the elimination of "non-malleable" commitment schemes. Instead, we only require commitments with the basic hiding and binding properties, whereas much more efficient schemes based on hash functions can be used (while still enjoying provable security).

Therefore, we compare both the overall computation and communication overhead between our MP-MAP and the SAS-GMA in Table. II. The communication overhead is evaluated in terms of number of bits transmitted/received. For the SAS-GMA protocol, we assume the use of a non-malleable commitment scheme from [MacKenzie and Yang 2004]. The constants $c_{1}$ and $c_{2}$ stands for the number of group elements (the length of each of them, $q$ is usually 1024-bits) in the commitment and decommitment, respectively. For example, for the DSA-based commitment scheme in [MacKenzie and Yang 2004], $c_{1}=2$ and $c_{2}=1$. In contrast, in the MP-MAP we use hash commitments and thus the length of a commitment value is the hash length $-n$. For instance, in SHA-256 $n=256$, and this is much smaller than $q$.

For the computation overhead, the main parts come from commitmen$\mathrm{t} /$ decommitment, hash function, and SAS computation. Common to both protocols is the use of a cryptographic hash function $H(\cdot)$ to hash an arbitrary long data $(\widehat{\mathcal{G}}|\widehat{\mathbf{c}}| \widehat{\mathbf{m}})$ to the length accepted by a universal hash (e.g., 256 bits) or digest function. The complexity for a cryptographic hash is based on the simple model in [Nguyen and Roscoe 2011], which is linear to both the input length and the output (or key) length. The $N^{2}$ factor is due to there are $N$ devices, and each device's hash input length is linear with $N$. For the commitment/decommitment, $c_{1}^{\prime}, c_{2}^{\prime}$ refer to the number of modular exponentiations required in their computations respectively. For the DSA-based commitment scheme in [MacKenzie and Yang 2004], $c_{1}^{\prime}=5, c_{2}^{\prime}=4$. For the SAS, the complexity of the digest function is even smaller than a cryptographic hash [Nguyen and Roscoe 2011], and is similar to a universal hash [Laur and Pasini 2008]. In summary, it can be seen that the MP-MAP is more efficient than SAS-GMA in terms of both computation and communication.

Finally, for our GDP protocol, the additional computation overhead to the MP-MAP is also small. It requires each sensor device to perform 3 modular exponentiations and $2 N-2$ modular multiplications for running the UDB key agreement protocol, and only one additional modular exponentiation for computing the individual key shared with controller. The computations for setting up the pairwise keys during the deployment phase rely on Galois field multiplications instead and are much more efficient. On the

\footnotetext{
${ }^{7}$ Construction based on hash function has also been proposed in [Laur and Nyberg 2006], but the security
} only remains as conjecture. 
other hand, the controller which is usually more powerful, needs to carry out $N+2$ modular exponentiations.

\subsection{Prototype Implementation}

We implemented GDP on a prototype sensor network platform consisting of 10 TmoteSky nodes, each with 8MHz TI-MSP430 microcontroller, 10KB RAM and 48KB Flash (ROM), and with TinyOS. We let one of the sensor nodes be the controller, which does not improve the performance of GDP protocol. For our experiments, we implement rounds 2-5 in Fig. 5 up to the computation of the group key and the individual keys. The counting step is omitted, by programming the IDs of the devices and the group size into them in advance.

We convert the Diffie-Hellman based group key agreement (UDB) to its elliptic curve cryptography (ECC) version, where the modular exponentiation and modular multiplication correspond to point multiplication and point addition, respectively. We use the primitive operations provided by TinyECC [Liu and Ning 2008], including point multiplication and point addition, with all optimizations enabled. To provide 80-bit key security, the finite field size used in ECC should be 160 bits. So we first compute 160bit group key and individual keys using ECC versions of the UDB and Diffie-Hellman key agreement, and then hash the keys. In [Liu and Ning 2008], for 160-bit ECC and with all optimizations enabled, the ECDH initialization time is reported to be $1.8 \mathrm{~s}$ on Micaz, while the key computation time is $2.1 \mathrm{~s}$. The required ROM and RAM sizes are $16 \mathrm{~KB}$ and $1.8 \mathrm{~KB}$ which are well below the capacities of a Tmote-Sky node. Since there are only 4 point multiplications in ECC version of the GDP protocol on sensor nodes, GDP is fairly practical to be implemented on low-end sensors.

For the hash commitment in GDP, we use a keyed hash (standard HMAC construction based on SHA-256), where the random nonce $r$ is used as the key, and $I D \mid m$ is the input data. For implementation of the digest function, since the software code for it is not available, we also employ the keyed hash instead, which is only for demonstration purposes ${ }^{8}$. We chunk the first $\ell$ bits of the keyed hash to be the SAS. Finally, we set $\ell=16$.

In Fig. 9, the experimental setup is depicted. Now we describe the protocol process and user experience in more detail. After all devices are powered on, all the devices display red LED by default. Then the user presses a reset button on the controller which broadcast a "reset" signal to all the others. After resetting, the user presses another button on the controller to initiate the protocol. The controller's last message in each round serves as synchronization signal, and different rounds are started/finished through state transitions on each device. In each round before the final one, the other sensors should display the same LED light pattern which indicates that they are synchronized. Before devices start to display SASes, they display green light for several seconds. The simultaneous LED blinking for SAS lasts for about 16 seconds; after that if the patterns are the same, the user presses a button on every device to confirm. Note that, in our implementation the synchronization signals sent out by the controller are quite reliable, since the sensor nodes are put close to each other which leads to very good channel conditions.

\subsection{Results}

In the following, we assume that $N_{\max }=10$. And we will show that for $N_{\max }=10$, it is practical for a human user to perform the initial trust establishment with little effort.

${ }^{8}$ This only increases the computation time since the digest function is more efficient than a hash [Nguyen and Roscoe 2008]. 


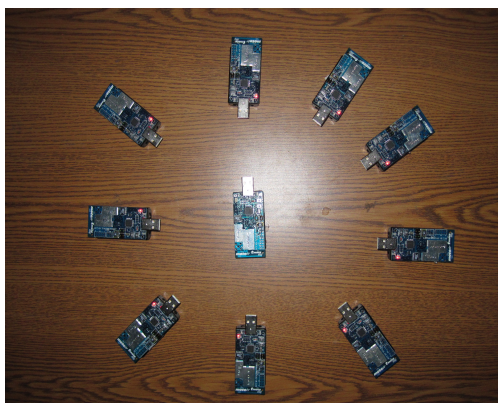

Fig. 9: Experimental setup with 10 devices. The central node is designated as the controller. All nodes are displaying synchronous LED blinking patterns. (c)2011, Ming Li)

\begin{tabular}{|c|c|c|c|c|c|}
\hline Decomposition & Commu. & Comput. & LED blink. & Idle & Total \\
\hline Time $(\mathrm{ms})$ & 409 & 11,005 & 15,360 & 3,187 & 29,961 \\
\hline Energy $(\mathrm{mJ})$ & 24.5 & 59.4 & 1,152 & 1.5 & $1,237.4$ \\
\hline
\end{tabular}

Table III: Decomposition of overhead of each sensor device in GDP $(N=10)$.

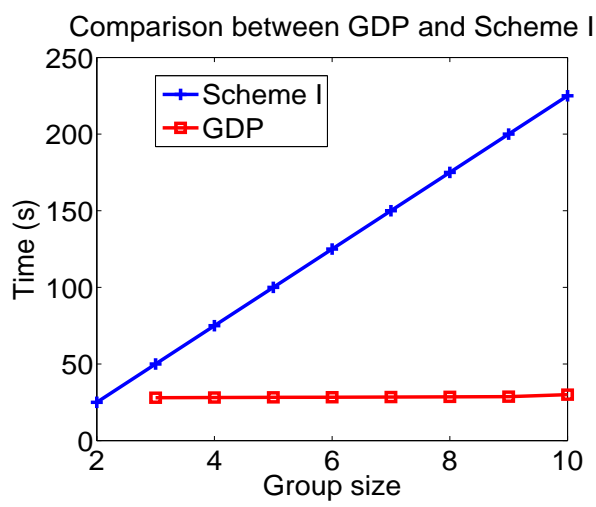

Fig. 10: Time for initial trust establishment.

For larger $N_{\max }$, specialized device could be used to aid the process such like the one in [Perković et al. 2011].

6.3.1. Time Required for Initial Trust Establishment. In our experiments, $N \leq N_{\max }$. So we plot the time for one GDP run $\left(T_{g d p}(N)\right)$ against the group size $N$ in Fig. 10 . It can be seen that $T_{g d p}$ is almost constant (increases linearly but very slowly) when $N$ increases. This is because all nodes display LED blinking patterns simultaneously, while the computations are quite fast. $T_{g d p}$ consists of time spent in computation $\left(T_{c p}\right)$, communication $\left(T_{c m}\right)$ and human interaction $\left(T_{I}\right)$. We then decompose $T_{g d p}$ in Table. III. For $\ell=16$ bits, $T_{I} \approx 16 \mathrm{~s}$ (one bit for $1 \mathrm{~s}$ ). Obviously, the LED blinking time takes a major portion, and then the computation time, and finally the communications. The idle time is needed for nodes to wait to receive all other's broadcasts in each round and to resolve collisions.

When $N>N_{\max }$, the number of subgroups $k=\left\lceil\frac{N-1}{N_{\max }-1}\right\rceil$. Then the total initial trust establishment time

$$
T_{g d p}(N) \approx(k-1) T_{g d p}\left(N_{\max }\right)+T_{g d p}\left(N-k\left(N_{\max }-1\right)\right),
$$




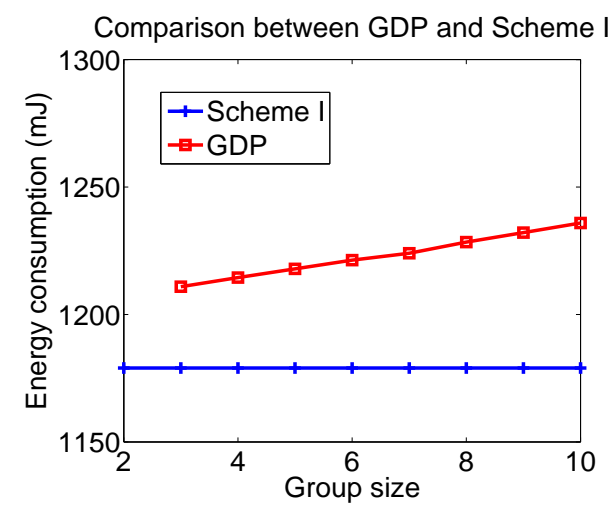

Fig. 11: Energy consumption per sensor node.

which increases linearly with $k$, and repeats the almost constant pattern when $N \leq$ $N_{\max }$. The above time can be approximated theoretically, based on the experimental values $T_{g d p}(N), N \leq N_{\max }$. For $N=20, T_{g d p} \approx 60$ s.

We also compare GDP with Scheme I, in which $T_{s c 1}(N)=(N-1) T_{s c 1}(2)$, where $T_{s c 1}(2)$ is the estimated time for pairwise device pairing. From Fig. 10, $T_{s c 1}(N)$ is linear with $N$. For $N=20$, this is 475s. Obviously, when $N \geq 3$ the time of GDP is far less than Scheme I, which is also the case for [Keoh et al. 2009] that uses one-by-one sensor association.

6.3.2. Energy Consumption. From the data sheet of Tmote [Tmo 2005], we obtain the normal voltage and current of the mote under different conditions, based on which we compute the energy consumption (EC). We plot the average EC for each sensor node in GDP against the group size $(N \leq 10)$ in Fig. 11, and compare it to the estimated EC of Scheme I (based on the EC break down for each primitive operation). The EC of GDP is a little higher than that of Scheme I, since it uses extra ECC point multiplication and addition operations. However, the difference is small (below $50 \mathrm{~mJ}$ ). Note that, for the controller, the EC of Scheme I is linear to $N$ which is much larger than that of GDP due to GDP's grouping mechanism.

Then we break down the EC of GDP in Table. III. It can be seen that the LED blinking takes major part in the EC, since its time is the longest and the required power is among the largest. Although the communication needs the largest power, it consumes the smallest energy since the time of it is quite small. Finally, note that the energy spent in computation is very small too, because the required power is small.

6.3.3. Usability and Security. GDP supports batch deployment. From the experiments, we found it is practical for a human to watch $n \leq 10$ LED blinking patterns simultaneously, when the nodes are put close to each other. The watch-and-compare is easy to follow, and differences can be identified with high probability. While MiB [Kuo et al. 2007] and KALwEN [Law et al. 2010] also achieve batch deployment, they require additional hardware (a faraday cage (FC), a keying device and a keying beacon). These devices add cost to the BAN and a FC is cumbersome to carry by the user. The SASGAKA [Laur and Pasini 2008] does not use additional device, however string comparison needs a user to remember strings which requires $N$ interactions. The results are summarized in Table. IV. We also compare with SPATE [Lin et al. 2009], a group message authentication protocol. It requires $N$ comparisons of "T-flags" for each user, while each comparison needs a few seconds, and the devices need to have screen/display. 


\begin{tabular}{|c|c|c|c|c|c|c|c|}
\hline & Comparison criteria & 鿖 & $\underset{z}{i}$ & $\underset{1}{Z}$ & 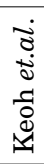 & 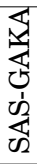 & 氙 \\
\hline 总 & $\begin{array}{c}\text { Key secrecy, authenticity } \\
\text { Key confirmation } \\
\text { Exclusiveness } \\
\text { Provable security }\end{array}$ & $\begin{array}{l}\sqrt{ } \\
\sqrt{ } \\
\sqrt{ } \\
\sqrt{ }\end{array}$ & $\begin{array}{l}\sqrt{ } \\
\sqrt{ } \\
\sqrt{ } \\
\times\end{array}$ & $\begin{array}{l}\sqrt{ } \\
\times \\
\sqrt{ } \\
\times\end{array}$ & $\begin{array}{l}\sqrt{ } \\
\sqrt{ } \\
\sqrt{ } \\
\sqrt{ }\end{array}$ & $\begin{array}{l}\sqrt{ } \\
\times \\
\times \\
\sqrt{ }\end{array}$ & $\begin{array}{l}\sqrt{ } \\
\times \\
\sqrt{ } \\
\times\end{array}$ \\
\hline 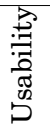 & $\begin{array}{c}\text { Fast batch deployment } \\
\text { Error-proof } \\
\text { \# of human interactions } \\
\text { Human effort }\end{array}$ & $\begin{array}{l}\sqrt{ } \\
\sqrt{ } \\
\ll \\
\mathrm{L}\end{array}$ & $\begin{array}{l}\sqrt{ } \\
\sqrt{ } \\
/ \\
M\end{array}$ & $\begin{array}{l}\sqrt{ } \\
\sqrt{ } \\
/ \\
M\end{array}$ & $\begin{array}{l}\times \\
\sqrt{ } \\
N \\
\mathrm{H}\end{array}$ & $\begin{array}{l}\sqrt{ } \\
\times \\
N \\
\mathbf{M}\end{array}$ & $\begin{array}{l}\sqrt{ } \\
\sqrt{ } \\
N \\
\text { M }\end{array}$ \\
\hline نे & $\begin{array}{c}\text { Requires NO PKI } \\
\text { No additional hardware } \\
\text { No interface on sensors } \\
\text { Involvement of PKC }\end{array}$ & $\begin{array}{l}\sqrt{ } \\
\sqrt{ } \\
\sqrt{ } \\
\mathrm{L}\end{array}$ & $\begin{array}{l}\sqrt{ } \\
\times \\
\sqrt{ } \\
\text { NA }\end{array}$ & $\begin{array}{c}\sqrt{ } \\
\times \\
\sqrt{ } \\
\text { NA }\end{array}$ & $\begin{array}{l}\times \\
\sqrt{ } \\
\sqrt{ } \\
M\end{array}$ & $\begin{array}{l}\sqrt{ } \\
\sqrt{ } \\
\times \\
H\end{array}$ & $\begin{array}{l}\sqrt{ } \\
\sqrt{ } \\
\times \\
\mathrm{L}\end{array}$ \\
\hline
\end{tabular}

Table IV: Comparison of GDP with related previous schemes. L: low; NA: none; M: medium; H: high.

Finally, from the security point of view, few of the compared protocols have formal security proofs. The SAS-GAKA is proven secure under a simulation-based security model, but it requires the use of non-malleable commitment schemes. The protocol in [Keoh et al. 2009] was proven secure using the Burrows-Abadi-Needham (BAN) logic, but the BAN logic is mainly suitable for proving traditional authentication protocols secure, which involves the existence of pre-shared secret keys between the parties.

\subsection{Efficiency of Key Management after Initial Trust Establishment}

6.4.1. Communication. The overhead for adding $N$ nodes is essentially the same as initial sensor association. The existing nodes do not need to perform extra communications. Revoking one node in subgroup $k$ requires $\# G+|\mathcal{G}(k)|-1$ unicasts of the controller, where $\# G$ is the number of subgroups. Our scheme is very efficient under group node leave, where the leaving nodes all belong to one subgroup or one cluster. If $m$ nodes leave in $\mathcal{G}(k)$, the controller only needs to send $\# G+\left|\mathcal{G}_{k}\right|-m$ messages. Clearly, if $\left|\mathcal{G}_{k}\right|=N_{\max }$, for single sensor leave/revocation there is an optimal value for $N_{\max }$, which equals to $\sqrt{N-1}$. For $N \leq 100$, this is smaller than 10 . Therefore, it provides a guideline to choose $N_{\max }$ for GDP.

6.4.2. Storage. If all the pairwise keys are stored along with the polynomial share, the size of the keys stored on each sensor node is: $2 \kappa+(N-1+t) \cdot \log p+n$ bits, where $\kappa$ is the bit length of symmetric key. If the sensors do not store the pairwise keys, then the minimum size of the keys is: $2 \kappa+t \cdot \log p+n$ bits. Assume $\kappa=80, \log p=80, t=$ $50, n=256$, the maximum size is $4416+79 N$ bits, while the minimum is 4416 bits. These numbers are well below 4KByte, the available RAM of Micaz.

\section{CONCLUSION}

In this paper, we address the problem of secure ad hoc initial trust establishment and key management in body area networks. We exploit the concept of "device pairing" and propose group device pairing (GDP), a novel solution that establishes an authenticated group consisting of low-end sensor devices and a controller, without relying on any pre-distributed secret information. An authenticated group key and individual keys are agreed upon using GDP, with the help of simultaneous and manual comparison of LED blinking patterns on all devices, which can be done within 30 seconds with enough security strength in practical applications. GDP helps the user of a BAN to 
visually make sure that the authenticated group only consists of those nodes that she wants to deploy and associate with the intended patient. The resulting initial key materials enable efficient key management after network deployment. We have proven the security of the proposed GDP and its two-party version (PDP) under standard security notions; especially we show the non-necessity of non-malleable commitment schemes. Efficiency analysis shows that GDP outperforms a previous group message authentication protocol, while experimental results show that GDP greatly reduces the total time and complexity of human effort, and being efficient both in communication and computation.

\section{ACKNOWLEDGMENTS}

We would like to thank Hanfei Zhao for his help with GDP's prototype implementation. We also thank Shahab Mirzadeh for his comments on the conference version of this paper. Finally, we thank the anonymous reviewers for their helpful comments.

\section{APPENDIX}

\section{A. SECURITY DEFINITION OF MAPS: MATCHING CONVERSATIONS}

In this section, we give a formal treatment of matching conversations [Bellare and Rogaway 1994], adapt it to group settings and deal with broadcast messages. First, if each participant $i \in \mathcal{G}$ has executed a local run (or partial run) $\mathcal{R}_{i}$, then we can interleave the events of all the local runs, arranging them in a single sequence, in many different ways. One of these sequences is a topological sort of $\left\{\mathcal{R}_{i}\right\}_{i \in \mathcal{G}}$ if, for all $i$, it preserves the order of events lying on the same $\mathcal{R}_{i}$. We use topological sorts to represent the notion of a proper matching up of transmission and reception events. When a protocol uses no broadcast, but only point-to-point messages, we can require that we can always place a matching transmission-reception pair next to each other. We will give the definitions first for the case without broadcast, and then loosen them for the case using broadcast, as is needed for our protocols.

Thus, we will say that the parties $i \in \mathcal{G}$ have jointly matching broadcast-free conversations in a family $\left\{\mathcal{R}_{i}\right\}_{i \in \mathcal{G}}$ of local runs if:

there is a topological sort of the transmission and reception events of all local runs $\mathcal{R}_{i}$, respecting the local ordering of each $\mathcal{R}_{i}$, such that

(1) every reception event $e_{1}$ immediately follows a transmission event $e_{0}$, and $e_{1}$ receives the same message sent at $e_{0}$; and

(2) vice versa, every transmission event $e_{0}$ immediately precedes a reception event $e_{1}$, and $e_{1}$ receives the same message sent at $e_{0}$.

Thus, if the parties have matching conversations, all messages transmitted by them will be received unaltered, i.e., authentically. This condition also implies that the same transmitted message is not delivered more than once, since only one reception can follow it immediately.

To generalize this notion to a group $\mathcal{G}$ with an uncorrupted subset $\mathcal{N} \subseteq \mathcal{G}$, we will suppose that associated with every reception $e_{1}$ along a local run $\mathcal{R}_{i}$ with $i \in \mathcal{N}$, there is an expected sender $j \in \mathcal{G}$. Likewise, associated with every transmission $e_{0}$ along a local run $\mathcal{R}_{i}$ with $i \in \mathcal{N}$, there is an expected recipient $j \in \mathcal{G}$. This is certainly the case with our protocols when the group $\mathcal{G}$ is known. Now, a set $\left\{\mathcal{R}_{i}\right\}_{i \in \mathcal{N}}$ of local runs for $i \in \mathcal{N}$ consists of jointly matching, broadcast-free matching conversations for the uncorrupted participants if:

there is a topological sort of the transmission and reception events of the local runs $\mathcal{R}_{i}$, respecting the local ordering of each $\mathcal{R}_{i}$, such that 
(1) for every reception event $e_{1}$, if the expected sender of $e_{1}$ is some $j \in$ $\mathcal{N}$, then $e_{1}$ immediately follows a transmission event $e_{0}$ on $\mathcal{R}_{j}$, and $e_{1}$ receives the same message sent at $e_{0}$; and

(2) vice versa, for every transmission event $e_{0}$, if the expected recipient of $e_{0}$ is some $j \in \mathcal{N}$, then $e_{0}$ immediately precedes a reception event $e_{1}$ on $\mathcal{R}_{j}$, and $e_{1}$ receives the same message sent at $e_{0}$.

Our previous definition without corruption, is equivalent to the case in which $\mathcal{N}=\mathcal{G}$, at least when the group is known, and each message makes its expected sender and expected recipient explicit.

To adapt our definition to the case with broadcast messages, we use a symbol * to represent the expected recipient of a broadcast message. We assume $* \notin \mathcal{G}$. The idea is that a message with expected recipient $*$ is broadcast and may be received by everyone. In this case, there may be several reception events, all following immediately after the transmission as a block. We assume here that $\mathcal{N}$ is non-empty. A set $\left\{\mathcal{R}_{i}\right\}_{i \in \mathcal{N}}$ of local runs for $i \in \mathcal{N}$ consists of jointly matching conversations for the uncorrupted participants if:

there is a topological sort of the transmission and reception events of the local runs $\mathcal{R}_{i}$, respecting the local ordering of each $\mathcal{R}_{i}$, such that

(1) for every transmission event $e_{0}, e_{0}$ immediately precedes a reception event $e_{1}$ on some $\mathcal{R}_{j}$, where the expected recipient of $e_{0}$ is either $j$ or $*$. If the expected recipient of $e_{0}$ is $j$, then $e_{1}$ is not followed by another reception event. Moreover, $e_{1}$ receives the same message sent at $e_{0}$.

(2) for every reception event $e_{1}$, if the expected sender of $e_{1}$ is some $j \in \mathcal{N}$, then $e_{1}$ immediately follows some event $e_{0}$, and $e_{0}$ involving the same message as $e_{0}$. If $e_{0}$ is a transmission event, then $e_{0}$ lies on $\mathcal{R}_{j}$.

\section{B. PROOF OF THEOREM 1}

Proof. Let the parties involved in a protocol run as $A$ and $B$. The first part of the security goal is obvious, so we only need to show that for any $T_{b}+2 T_{h}+O(1)$-time adversary $\mathcal{A}$, whenever the assumptions of the theorem hold, its deception probability $\operatorname{Adv}_{\Pi}(\mathcal{A})$ is no larger than $\max \left\{\epsilon_{u}, \epsilon_{r}, \epsilon_{h}^{2}\right\}+\max \left\{\epsilon_{r}, \epsilon_{b}\right\}$. We first denote the event " $\mathcal{A}$ succeeds in deception" as $S$, where

$$
S=\left\{S_{1} \wedge S_{2}\right\} \triangleq\left\{\text { Both-accept } \wedge \text { No-matching }{ }^{\mathcal{A}}\right\},
$$

where No-matching ${ }^{\mathcal{A}}$ refers to the event that $A$ and $B$ do no have matching conversations. Note that, in order for both of them to accept, they need to successfully verify the SASes are equal (Fig. 3), and they must not abort during the protocol. It is easy to see that $\operatorname{Adv}_{\Pi}(\mathcal{A})=\operatorname{Pr}[S]$.

Next we analyze $\operatorname{Pr}[S]$. First we define "view ${ }_{i}$ " as the ordered set consisting of all the messages received by device $i$ in the round $2\left(\right.$ view $_{A}=\left\{m_{A}, \widehat{m}_{B}, c_{A}, \widehat{c}_{B}\right\}$, and view ${ }_{B}=$ $\left.\left\{\widehat{m}_{A}, m_{B}, \widehat{c}_{A}, c_{B}\right\}\right)$.

We will use the following lemma to continue our proof.

LEMMA 1. In the TP-MAP, if event $S_{2}$ happens (No-matching ${ }^{\mathcal{A}}$ ), then either view $w_{A} \neq$ view $_{B}$, or otherwise, A and $B$ will accept with probability $\epsilon_{b}$.

The above is straightforward to prove. To see that, notice if view ${ }_{A}=$ view $_{B}$, to create no-matching conversations the adversary must break the binding property of hash commitments (i.e., to find a different $d$ for the same $m$ and $c$ values), and the probability of success is no larger than $\epsilon_{b}$. Thus, we can define an event $E \triangleq\left\{\operatorname{view}_{A} \neq \operatorname{view}_{B}\right\}$. 


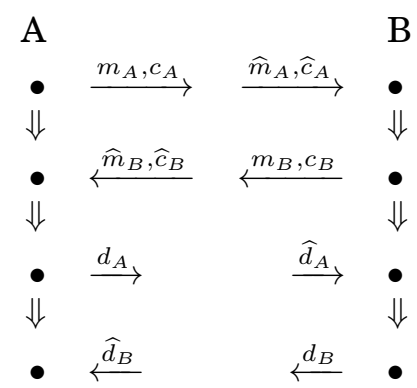

Fig. 12: Diagram for a partial execution of the protocol in the TP-MAP for our PDP. Note that, here $m_{i}$ contains $I D_{i}$ and the message to be authenticated.

Observe that, by the total probability principle, we have

$$
\begin{aligned}
\operatorname{Pr}[S] & =\operatorname{Pr}[S \mid E] P[E]+\operatorname{Pr}[S \mid \bar{E}] P[\bar{E}] \\
& \stackrel{(1)}{\leq} \operatorname{Pr}[S \mid E]+\operatorname{Pr}[S \mid \bar{E}] \\
& \stackrel{(2)}{=} \operatorname{Pr}\left[S_{1} \mid E\right]+\epsilon_{b} .
\end{aligned}
$$

where (1) follows from $\operatorname{Pr}[E], \operatorname{Pr}[\bar{E}] \leq 1$, and (2) follows from Lemma. 1 and the fact that $E$ implies $S_{2}$ (no-matching conversations).

Therefore, next we focus on the case that event $E$ happens, and assume $\mathcal{A}$ does not break the binding property of hash commitments. There are two cases for view $A \neq$ view $_{B}$ that deserves discussion. (1) $\widehat{\mathbf{m}}_{A} \neq \widehat{\mathbf{m}}_{B}$ but $\widehat{\mathbf{c}}_{A}=\widehat{\mathbf{c}}_{B}$. This again corresponds to a double opening of the hash commitment, and the probability the adversary will succeed in this way is bounded by $\epsilon_{b}$. (2) $\widehat{\mathbf{c}}_{A} \neq \widehat{\mathbf{c}}_{B}$. We have the following lemma for this case.

LEMMA 2. In the TP-MAP, given $\widehat{\boldsymbol{c}}_{A} \neq \widehat{\boldsymbol{c}}_{B}$, for any $T_{h}+O(1)$ time adversary that does not break the binding of hash commitments, $\operatorname{Pr}\left[S_{1} \mid E\right] \leq \max \left\{\epsilon_{u}, \epsilon_{r}\right\}+\epsilon_{h}$.

PROOF. To be clear, consider the diagram for a partial execution (first four moves) of the protocol ${ }^{9}$ in Fig. 12. The black dots stand for the decision points of each party's run (also called a strand), while down arrows represent parties' internal state transitions. The blank parts between two strands indicate a party' sent messages can be manipulated by any outsider adversary before they are received by the other party. The first two moves consist the first round, and the second and fourth moves consist the second round.

First let us assume the adversary $\mathcal{A}$ does not break the hiding property of hash commitments in the first round (this strategy is denote as $H$ ). This does not preclude the following three general strategies - (a) $\mathcal{A}$ can simply relay a message truthfully; (b) $\mathcal{A}$ can create a new $\widehat{c}_{A}$ or $\widehat{c}_{B}$ using $\widehat{r}_{A}$ or $\widehat{r}_{B}$ values of her own choice, but are independent of $r_{A}$ and $r_{B}$; (c) create "related" $\widehat{c}_{A}$ and $\widehat{c}_{B}$ committing to unknown $\widehat{r}_{A}$ and $\widehat{r}_{B}$, that are "correlated" to $r_{A}$ and/or $r_{B}$, respectively, after seeing $c_{A}$ and $c_{B}$ (malleability). (although she does not know the latter two). The "correlation" $(\sim)$ between those $\mathbf{r}$ variables could mean anything except their independence. But here, it must have a constraint - the variables (regarded as bit strings) have the same length, otherwise it does not make sense. So relations like string concatenations are excluded. The

${ }^{9}$ Here we adopt the protocol representation in strand spaces proposed by [Guttman 2011]. 
simplest relation is equality; however, relaying is the same with (a), while the replay attack (copying $c_{A}$ as $\widehat{c}_{B}$ ) is prevented since the commitments have included sender $I D$ in it, and the replay will not pass the verification of $A$.

Since $\mathcal{A}$ does not break the binding property of hash commitments, the digest keys are bound to the commitments. So we can focus on the commitment round only. In order to succeed, $\mathcal{A}$ must create $\widehat{c}_{A}$ and $\widehat{c}_{B}$ such that sas ${ }_{A}=\operatorname{sas}_{B}$. For $\widehat{c}_{A}$, if $\mathcal{A}$ chooses strategy (a) and let $\widehat{c}_{A}=c_{A}$, then $r_{A}$ and $r_{B}$ are independent since they are randomly generated by $A$ and $B$, respectively; if $\mathcal{A}$ chooses (b) or (c), due to the precedence $\widehat{c}_{A} \prec$ $c_{B}, \widehat{r}_{A}$ will still be independent from $r_{B}$, which is unknown by $\mathcal{A}$.

As $\widehat{c}_{B}$ is the last message $\mathcal{A}$ can send, it must obtain a corresponding $\widehat{r}_{B}$ such that sas $_{A}=$ sas $_{B}$. Note that, $\mathcal{A}$ cannot simply relay both $c_{A}$ and $c_{B}$. Next we discuss the case when $\widehat{c}_{A} \neq c_{A}$.

$-\mathcal{A}$ can choose strategy (b) to construct any of $\widehat{c}_{A}$. No matter how $\widehat{c}_{B}$ is constructed, $\widehat{r}_{A}$ must be independent from both $r_{A}$ and $r_{B}$ which are unknown to $\mathcal{A}$. So according to the key-based uniformity property of digest function, the probability of finding $\widehat{r}_{A}$ such that $\mathcal{H}\left(\widehat{\mathbf{m}}_{A}, r_{A} \oplus \widehat{r}_{B}\right)=y=\mathcal{H}\left(\widehat{\mathbf{m}}_{B}, \widehat{r}_{A} \oplus r_{B}\right)$ is smaller or equal to $\epsilon_{u}$, where $y$ is a fixed number.

- So $\mathcal{A}$ can choose strategy (a) or (c) for $\widehat{c}_{A}$ and $\widehat{c}_{B}$. According to our definitions, we have $\widehat{r}_{A} \sim r_{A}$ and $\widehat{r}_{B} \sim r_{B}$ or $\widehat{r}_{B} \sim r_{A}$. W.l.o.g., suppose $\widehat{r}_{A}=r_{A} \oplus \theta_{1}$ and $\widehat{r}_{B}=r_{B} \oplus \theta_{2}$ or $\widehat{r}_{B}=r_{A} \oplus \theta_{3}$. In the first case, we have $\operatorname{Pr}\left[\mathcal{H}\left(\widehat{\mathbf{m}}_{A}, r_{A} \oplus r_{B} \oplus \theta_{2}\right)=\mathcal{H}\left(\widehat{\mathbf{m}}_{B}, r_{A} \oplus r_{B} \oplus \theta_{1}\right)\right] \leq$ $\epsilon_{r}$ according to the no uniform compensation property of digest function, where $\theta=$ $\theta_{1} \oplus \theta_{2}$. In the second case, we have $\operatorname{Pr}\left[\mathcal{H}\left(\widehat{\mathbf{m}}_{A}, \theta_{3}\right)=\mathcal{H}\left(\widehat{\mathbf{m}}_{B}, r_{A} \oplus r_{B} \oplus \theta_{1}\right)=y\right] \leq \epsilon_{u}$, since $y$ is a fixed (unknown) number.

For the case $\widehat{c}_{A}=c_{A}$, it can be shown similarly that $\mathcal{A}$ 's probability to succeed is no larger than $\max \left\{\epsilon_{u}, \epsilon_{r}\right\}$. Combining the above, we get $\operatorname{Pr}\left[S_{1} \mid E\right] \leq \max \left\{\epsilon_{u}, \epsilon_{r}\right\}$ for any $O(1)$-time adversary that does not break hiding of hash commitments.

Second, if the hiding property of any hash commitment is broken, $\mathcal{A}$ 's probability of success is bounded by $\epsilon_{h}$ for any $T_{h}$ time $\mathcal{A}$. So the Lemma is proved.

Using Eq. (5) and Lemma 2, we get

$$
\operatorname{Pr}[S] \leq \max \left\{\epsilon_{u}, \epsilon_{r}\right\}+\epsilon_{h}+2 \epsilon_{b},
$$

for any adversary $\mathcal{A}$ that runs in $2 T_{b}+T_{h}+O(1)$ time.

\section{PROOF OF THEOREM 2}

Proof. First we define "view" ${ }^{\prime}$ as the set of information exchanged in the second round, which is the ordered set consisting of all the messages $\left(\widehat{\mathbf{m}}_{i}, \widehat{\mathbf{c}}_{i}\right)$, i.e., $\left(\left\{\widehat{I D}_{j}\left|\widehat{X}_{j}\right| \widehat{Y}_{j}\right\},\left\{\widehat{c}_{j}\right\}\right), j \in \mathcal{G}_{i}$ received by device $i$ in the round 2 .

In this proof, we use $\mathcal{G}$ to denote the true group of legitimate devices (perceived by human user), and $\mathcal{N}$ to denote the subset of non-compromised devices in $\mathcal{G}$. Similar to TP-MAP, we define

$$
S=\left\{S_{1} \wedge S_{2}\right\} \triangleq\left\{\text { All-accept } \wedge \text { No-matching }{ }^{\mathcal{A}}\right\},
$$

where All-accept means all devices in $\mathcal{N}$ accept, while No-matching ${ }^{\mathcal{A}}$ refers to the event that there exist $i, j \in \mathcal{N}$ such that their conversations do not match. We will use the following lemma to continue our proof.

LEMMA 3. If event $S_{2}$ happens (No-matching ${ }^{\mathcal{A}}$ ), then either $\exists i, j \in \mathcal{N}$ such that view $_{i} \neq$ view $_{j}$, or otherwise, all the devices in $\mathcal{N}$ will accept with probability $\epsilon_{b}$.

The argument for this Lemma is similar to that of Lemma. 1. 
Define event $E \triangleq\left\{\exists i, j \in \mathcal{N}\right.$, s.t.view $\neq$ view $\left._{j}\right\}$, we have the following:

$$
\begin{aligned}
\operatorname{Pr}[S] & =\operatorname{Pr}[S \mid E] P[E]+\operatorname{Pr}[S \mid \bar{E}] P[\bar{E}] \\
& \stackrel{(1)}{\leq} \operatorname{Pr}[S \mid E]+\operatorname{Pr}[S \mid \bar{E}] \\
& \stackrel{(2)}{=} \operatorname{Pr}\left[S_{1} \mid E\right]+\epsilon_{b} .
\end{aligned}
$$

The second equation follows from Lemma. 3 and the fact that $E$ implies $S_{2}$ (nomatching conversations).

Next, we use the following bound to constrain our discussion to the scenario that all pair of non-compromised devices' SASes match except one pair $N$ and $i$ (event denoted as $\left.S_{1-N i}\right), \forall i \in \mathcal{N} \backslash N$, while $N$ and $i$ 's views do not equal. Applying the probability product rule, we get:

$$
\begin{aligned}
\operatorname{Pr}\left[S_{1} \mid E\right] & =\frac{\operatorname{Pr}\left[S_{1}, E\right]}{\operatorname{Pr}[E]} \\
& \leq \frac{\operatorname{Pr}\left[S_{1}, E\right]}{\operatorname{Pr}\left[E, S_{1-N i}\right]} \\
& =\operatorname{Pr}\left[S_{N i} \mid E, S_{1-N i}\right],
\end{aligned}
$$

where $S_{N i} \triangleq\left\{\right.$ sas $_{N}=$ sas $\left._{i}\right\}$, because $S_{1}=\left\{S_{N i}, S_{1-N i}\right\}$. Also, note that event $E$ implies there must exist some $i$ such that view ${ }_{i} \neq$ view $_{N}$ where $N$ is the controller, we can further decompose $\operatorname{Pr}\left[S_{N i} \mid E, S_{1-N i}\right]$ into two cases for $i$ and $N$, i.e., $\left|\mathcal{G}_{i}\right|=N$ or $\left|\mathcal{G}_{i}\right| \neq N$ (note that $N=\left|\mathcal{G}_{N}\right|$, otherwise $M_{N}$ will not accept).

Connecting the above, we thus have the following bound on $\operatorname{Pr}[S]$ :

$$
\operatorname{Pr}\left[S_{1} \mid E\right] \leq \max \left\{\begin{array}{l}
\operatorname{Pr}\left[S_{N i}\left|E, S_{1-N i},\right| \mathcal{G}_{i} \mid=N\right], \\
\operatorname{Pr}\left[S_{N i}\left|E, S_{1-N i},\right| \mathcal{G}_{i} \mid \neq N\right] .
\end{array}\right.
$$

It remains to show that the probabilities on the right hand side are upper bounded by $\max \left\{\epsilon_{u}, \epsilon_{r}\right\}+\epsilon_{h}+\epsilon_{b}$. We first focus on the case of $E, S_{1-N i},\left|\mathcal{G}_{i}\right|=N$.

There are two cases for view view $_{N}$ that deserves discussion. (1) $\widehat{\mathbf{m}}_{i} \neq \widehat{\mathbf{m}}_{N}$ but $\widehat{\mathbf{c}}_{i}=$ $\widehat{\mathbf{c}}_{N}$. This corresponds to a double opening of the hash commitment, and the probability the adversary will succeed in this way is bounded by $\epsilon_{b}$. (2) $\widehat{\mathbf{c}}_{i} \neq \widehat{\mathbf{c}}_{N}$. Here we need to consider two cases: $\mathcal{N}=\mathcal{G}$ (no compromised insiders) or $\mathcal{N} \varsubsetneqq \mathcal{G}$ (some devices are compromised). We first discuss the former case. We have the following lemma, whose proof is shown later.

LEMMA 4. In the MP-MAP, given $\widehat{\boldsymbol{c}}_{i} \neq \widehat{\boldsymbol{c}}_{N}$, for any $T_{h}$ time adversary that does not break the binding of hash commitments, $\operatorname{Pr}\left[S_{N i}\left|E, S_{1-N i},\right| \mathcal{G}_{i} \mid=N\right] \leq \max \left\{\epsilon_{u}, \epsilon_{r}\right\}+\epsilon_{h}$.

For the case of $E, S_{1-N i},\left|\mathcal{G}_{i}\right| \neq N$, using a similar analysis to the proof of Lemma. 4, the same conclusion can be drawn. Note that since $\left|\mathcal{G}_{i}\right| \neq\left|\mathcal{G}_{N}\right|$, in the SASes of $i$ and $N$, respectively, their data input parts of the digest function will never equal to each other even if $\widehat{\mathbf{c}}_{i}=\widehat{\mathbf{c}}_{N}$ and $\widehat{\mathbf{m}}_{i}=\widehat{\mathbf{m}}_{N}$, while this does not affect the result. In fact, this is why we should include all the protocol transcript into the SAS digest.

From the above, we know that the right hand side of Eq. (10) is bounded by $\max \left\{\epsilon_{u}, \epsilon_{r}\right\}+\epsilon_{h}+\epsilon_{b}$ for a $T_{b}+T_{h}$ time adversary. Summing up Eqs. (8), (9) and (10), we get $\operatorname{Pr}[S] \leq \max \left\{\epsilon_{u}, \epsilon_{r}\right\}+\epsilon_{h}+2 \epsilon_{b}$ for a $2 T_{b}+T_{h}$ time adversary. 


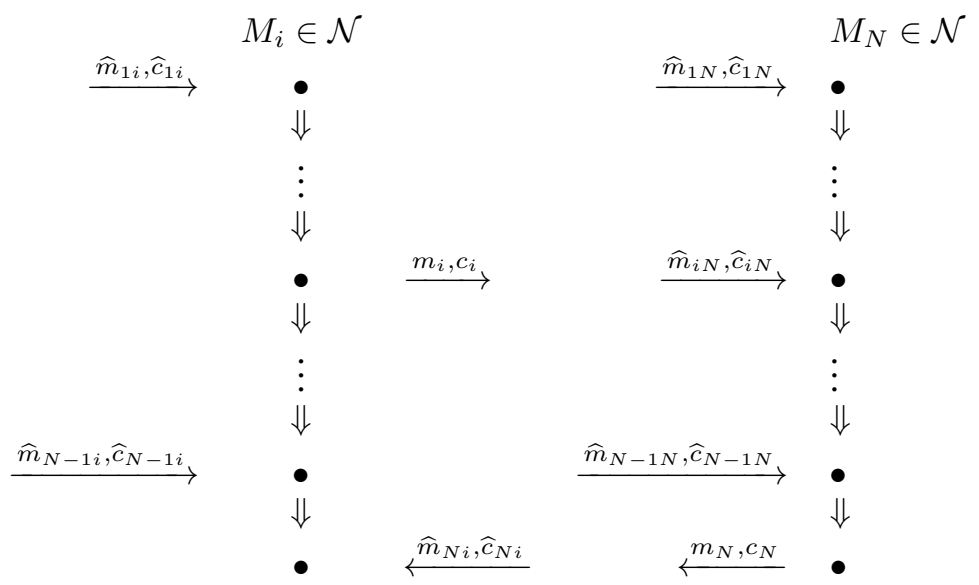

Fig. 13: Simplified diagram for a partial execution concerning devices $i$ and $N$ in round 2 of protocol MP-MAP. $m$ stands for the message to be authenticated.

\section{Proof of Lemma. 4.}

Proof. Consider the simplified diagram in Fig. 13. When $\mathcal{N}=\mathcal{G}$, our proof strategy is to show that, if $\mathcal{A}$ does not break the hiding of any hash commitments, its probability of success will be bounded by $\max \left\{\epsilon_{u}, \epsilon_{r}\right\}$. On the other hand, if any hash commitment's hiding is broken, $\mathcal{A}$ 's probability of success is bounded by $\epsilon_{h}$ for any $T_{h}$ time $\mathcal{A}$.

Then we focus on proving the first statement above. Adversary $\mathcal{A}$ can generate commitments $\widehat{c}_{1 i}, \ldots, \widehat{c}_{i-1 i}, \widehat{c}_{i+1 i}, \ldots, \widehat{c}_{N i}$ and $\widehat{c}_{1 N}, \ldots, \widehat{c}_{N-1 N}$ in arbitrary ways. It can either simply relay the original commitments sent by honest parties (without knowing the underlying $r$ values), or construct new commitments using its own $\widehat{r}$ values, or create commitment $\widehat{c}_{j^{\prime} i^{\prime}}$ that is related to any $c_{j}, j \in \mathcal{G}$ while does not know $\widehat{r}_{j^{\prime} i^{\prime}}$, where either $j=j^{\prime}$ or $j \prec j^{\prime}$.

Since the last message $\mathcal{A}$ can inject/modify is $\widehat{c}_{N i}$, we can focus on how $\mathcal{A}$ can compute it to make sas ${ }_{i}=$ sas $_{N}$. The SASes are in the following forms: sas ${ }_{i}=\mathcal{H}\left(\eta_{i}, \widehat{r}_{1 i} \oplus \ldots \oplus r_{i} \oplus\right.$ $\left.\ldots \oplus \widehat{r}_{N i}\right)$ (denoting $\eta_{i}$ as the data inputs); and similarly, sas ${ }_{N}=\mathcal{H}\left(\eta_{N}, \widehat{r}_{1 N} \oplus \ldots \oplus \widehat{r}_{N-1 N} \oplus\right.$ $\left.r_{N}\right)$. In the above, both $r_{i}$ and $r_{N}$ are unknown to $\mathcal{A}$, and since $\widehat{\mathbf{c}}_{i} \neq \widehat{\mathbf{c}}_{N}, \eta_{i} \neq \eta_{N}$.

Note that, all the $\widehat{c}_{1 N}, \ldots, \widehat{c}_{N-1 N}$ must be created before $c_{N}$ is sent out due to the message ordering, so $\widehat{r}_{1 N}, \ldots, \widehat{r}_{N-1 N}$ must be independent of $r_{N}$ which is unknown to $\mathcal{A}$. We have two cases:

- If $\mathcal{A}$ relays $c_{N}$ to $i$ and $\widehat{r}_{N i}=r_{N}$, since $\widehat{c}_{1 i}, \ldots, \widehat{c}_{i-1 i}, \widehat{c}_{i+1 i}, \ldots, \widehat{c}_{N-1 i} \prec \widehat{c}_{N i}, \widehat{r}_{1 i}, \ldots, \widehat{r}_{N-1 i}$ must be all independent with $r_{N}$. Thus, sas sa $_{i}=\mathcal{H}\left(\eta_{i}, \theta_{i} \oplus r_{N}\right)$, where $\theta_{i}=\widehat{r}_{1 i} \oplus$ $\ldots \oplus r_{i} \oplus \ldots \oplus \widehat{r}_{N-1 i}$ is independent of $r_{N}$, and sas ${ }_{N}=\mathcal{H}\left(\eta_{N}, \theta_{N} \oplus r_{N}\right)$, where $\theta_{N}=$ $\widehat{r}_{1 N} \oplus \ldots \oplus r_{N} \oplus \ldots \oplus \widehat{r}_{N-1 N}$ is independent of $r_{N}$. In the above, no matter whether $\theta_{i}$ is known to $\mathcal{A}$ or not, it is a fixed number when $\mathcal{A}$ relays $r_{N}$ to $i$, and the same is true for $\theta_{N}$. In addition, $\eta_{i} \neq \eta_{N}$. So according to the no uniform compensation property of digest function, $\operatorname{Pr}\left[\mathcal{H}\left(\eta_{i}, \theta_{i} \oplus r_{N}\right)=\mathcal{H}\left(\eta_{N}, \theta_{N} \oplus r_{N}\right)\right] \leq \epsilon_{r}$, and $\theta=\theta_{i} \oplus \theta_{N}$.

- If $\widehat{c}_{N i}$ is created by $\mathcal{A}$ using other strategies. Because $\mathcal{A}$ is free to create related commitments to $c_{i}$ after seeing $c_{i}$, and is also free to create its own commitments, it could make $\theta_{i} \oplus \widehat{r}_{N i}$ equal to a number $\theta_{i}^{\prime}$ it knows (otherwise, there will be an unknown factor $r_{j}$ in $\widehat{r}_{1 i} \oplus \ldots \oplus \widehat{r}_{N i}$, which reduces to the same case as above). Also, sas $_{N}$ is fixed when $\mathcal{A}$ sends $\widehat{r}_{N i}$ to $i$. So the key-based uniformity property of digest function applies and $\operatorname{Pr}\left[\mathcal{H}\left(\eta_{i}, \theta_{i}^{\prime}\right)=\mathcal{H}\left(\eta_{N}, \theta_{N} \oplus r_{N}\right)\right] \leq \epsilon_{u}$. 
Finally, for the scenario with compromised devices $(\mathcal{N} \varsubsetneqq \mathcal{G})$, the only additional information to $\mathcal{A}$ is the internal $r_{j}$ values for $j \in \mathcal{G} \backslash \mathcal{N}, j \neq i, j \neq N$. It is easy to see that the above proof still holds as long as $i$ and $N$ are not compromised $\left(r_{i}, r_{N}\right.$ are not known by $\mathcal{A}$ ).

\section{REFERENCES}

2005. Tmote-Sky Product Description Key Features. www.bandwavetech.com/download/tmote-skydatasheet.pdf.

Alliance, W. 2006. Association Models Supplement to the Certified Wireless Universal Serial Bus Specification. Revision 1, 3.

Ateniese, G., Steiner, M., And Tsudik, G. 2000. New multiparty authentication services and key agreement protocols. IEEE JSAC 18, 4, 628-639.

Balfanz, D., Smetters, D. K., Stewart, P., AND Wong, H. C. 2002. Talking to strangers: authentication in ad-hoc wireless networks. In NDSS '02.

Bellare, M., CANetti, R., AND KRAWCZyK, H. 1998. A modular approach to the design and analysis of authentication and key exchange protocols (extended abstract). In Proceedings of the thirtieth annual ACM symposium on Theory of computing. ACM, 419-428.

Bellare, M. AND Rogaway, P. 1994. Entity authentication and key distribution. In Advances in CryptologyCRYPTO93. Springer, 232-249.

Blundo, C., Santis, A. D., Herzberg, A., Kutten, S., Vaccaro, U., And Yung, M. 1993. Perfectlysecure key distribution for dynamic conferences. In CRYPTO '92. Springer-Verlag, 471-486.

Cagalu, M., Capkun, S., AND Hubaux, J.-P. 2006. Key agreement in peer-to-peer wireless networks. Proceedings of the IEEE 94, 2, 467-478.

Chan, H., Perrig, A., AND Song, D. 2003. Random key predistribution schemes for sensor networks. In IEEE $S$ \& $P$ '03. 197.

Chen, C.-H. O., Chen, C.-W., Kuo, C., Lai, Y.-H., McCune, J. M., Studer, A., Perrig, A., Yang, B.-Y., AND WU, T.-C. 2008. Gangs: gather, authenticate 'n group securely. In MobiCom '08. 92-103.

Di Pietro, R., Mancini, L., AND Mei, A. 2003. Random key-assignment for secure wireless sensor networks. In Proceedings of the 1st ACM workshop on Security of ad hoc and sensor networks. ACM, 62-71.

Du, W., Deng, J., Han, Y., VArshney, P., KatZ, J., AND Khalili, A. 2005. A pairwise key predistribution scheme for wireless sensor networks. ACM Transactions on Information and System Security (TISSEC) 8, 2, 228-258.

DutTa, R. AND BARUA, R. 2008. Provably secure constant round contributory group key agreement in dynamic setting. IEEE Trans. on Inf. Theory 54, 5, 2007-2025.

Eschenauer, L. AND Gligor, V. D. 2002. A key-management scheme for distributed sensor networks. In CCS '02. 41-47.

Goodrich, M. T., Sirivianos, M., Solis, J., Tsudik, G., AND UzUn, E. 2006. Loud and clear: Humanverifiable authentication based on audio. In In IEEE ICDCS 2006. 10.

GutTman, J. 2011. Shapes: Surveying crypto protocol runs. In Formal Models and Techniques for Analyzing Security Protocols, Cryptology and Information Security Series. IOS Press.

HALEVI, S. AND MiCALI, S. 1996. Practical and provably-secure commitment schemes from collision-free hashing. In Advances in CryptologyCRYPTO96. Springer, 201-215.

Hanson, M., Powell, H., Barth, A., Ringgenberg, K., Calhoun, B., Aylor, J., And Lach, J. 2009. Body area sensor networks: Challenges and opportunities. Computer 42, 1, 58-65.

Jana, S., Premnath, S., Clark, M., Kasera, S., Patwari, N., And Krishnamurthy, S. 2009. On the effectiveness of secret key extraction from wireless signal strength in real environments. In Proceedings of the 15th annual international conference on Mobile computing and networking. ACM, 321-332.

Jovanov, E., Milenkovic, A., OtTo, C., AND De Groen, P. C. 2005. A wireless body area network of intelligent motion sensors for computer assisted physical rehabilitation. J Neuroengineering Rehabil 2, 1.

KEOH, S. L., LUPU, E., AND Sloman, M. 2009. Securing body sensor networks: Sensor association and key management. IEEE PerCom '09, 1-6.

Kumar, A., Saxena, N., Tsudik, G., And Uzun, E. 2009. Caveat eptor: A comparative study of secure device pairing methods. IEEE PerCom '09, 1-10.

Kuo, C., Luk, M., Negi, R., AND PERriG, A. 2007. Message-in-a-bottle: user-friendly and secure key deployment for sensor nodes. In SenSys '07. 233-246.

LAMPORT, L. 1981. Password authentication with insecure communication. Commun. ACM 24, 11, 770-772. 
LAUR, S., Asokan, N., AND Nyberg, K. 2005. Efficient mutual data authentication using manually authenticated strings. In Cryptology and Network Security. Springer, 90-107.

LAUR, S. AND NyBERG, K. 2006. Efficient mutual data authentication using manually authenticated strings. Cryptology and Network Security, 90-107.

LAUR, S. AND PASInI, S. 2008. SAS-Based Group Authentication and Key Agreement Protocols. In Public Key Cryptography - PKC '08. LNCS. 197-213.

LAUR, S. AND PASINI, S. 2009. User-aided data authentication. International Journal of Security and Networks 4, 1, 69-86.

Law, Y., Moniava, G., Gong, Z., Hartel, P., And Palaniswami, M. 2010. Kalwen: A new practical and interoperable key management scheme for body sensor networks. Security and Communication Networks.

LI, M., LOU, W., AND REN, K. 2010a. Data security and privacy in wireless body area networks. Wireless Communications, IEEE 17, 1, 51-58.

LI, M., LOU, W., AND REN, K. 2010b. Secure device pairing. in Encyclopedia of Cryptography and Security (2nd Ed.).

LI, M., YU, S., LOU, W., AND REN, K. 2010. Group device pairing based secure sensor association and key management for body area networks. In INFOCOM, 2010 Proceedings IEEE. IEEE, 1-9.

Lin, Y.-H., Studer, A., Hsiao, H.-C., McCune, J. M., Wang, K.-H., Krohn, M., Lin, P.-L., Perrig, A., SUN, H.-M., AND YANG, B.-Y. 2009. Spate: small-group pki-less authenticated trust establishment. In Mobisys '09. 1-14.

LiU, A. AND NiNG, P. 2008. Tinyecc: A configurable library for elliptic curve cryptography in wireless sensor networks. In IPSN '08. 245-256.

LIU, D. AND NING, P. 2003. Establishing pairwise keys in distributed sensor networks. In CCS '03. 52-61.

LIU, D., NING, P., AND DU, W. 2008. Group-based key predistribution for wireless sensor networks. ACM Transactions on Sensor Networks (TOSN) 4, 2, 1-30.

Lorincz, K., Malan, D., Fulford-Jones, T., Nawoj, A., Clavel, A., Shnayder, V., Mainland, G., WELSH, M., AND MOULTON, S. 2004. Sensor networks for emergency response: challenges and opportunities. IEEE Pervasive Computing 3, 4, 16-23.

MACKEnZIE, P. AND YANG, K. 2004. On simulation-sound trapdoor commitments. In Advances in Cryptology-EUROCRYPT 2004. Springer, 382-400.

Malan, D., Welsh, M., AND Smith, M. 2004. A public-key infrastructure for key distribution in tinyos based on elliptic curve cryptography. In IEEE SECON 2004. 71-80.

MALASRI, K. AND WANG, L. 2007. Addressing security in medical sensor networks. In HealthNet '07. 7-12.

Mathur, S., Trappe, W., Mandayam, N., Ye, C., AND RezniK, A. 2008. Radio-telepathy: extracting a secret key from an unauthenticated wireless channel. In Proceedings of the 14th ACM international conference on Mobile computing and networking. ACM, 128-139.

McCune, J. M., Perrig, A., AND Reiter, M. K. 2005. Seeing-is-believing: Using camera phones for human-verifiable authentication. In IEEE $S$ \& P. 110-124.

Morchon, O., BALdus, H., AND SANCHEZ, D. 2006. Resource-efficient security for medical body sensor networks. In $B S N$ '06. 83.

NGUYen, L. AND Roscoe, A. 2008. Authenticating ad hoc networks by comparison of short digests. Information and Computation 206, 2-4, 250-271.

NGUYen, L. AND Roscoe, A. 2011. Authentication protocols based on low-bandwidth unspoofable channels: a comparative survey. Journal of Computer Security 19, 1, 139-201.

Nithyanand, R., SAXena, N., Tsudik, G., AND UZun, E. 2010. Groupthink: Usability of secure group association for wireless devices. In Proceedings of the 12th ACM international conference on Ubiquitous computing. ACM, 331-340.

Pasini, S. And Vaudenay, S. 2006. SAS-based Authenticated Key Agreement. In Public Key Cryptography - $P K C$ '06. LNCS Series, vol. 3958. $395-409$.

PASS, R. 2003. On deniability in the common reference string and random oracle model. Advances in Cryptology-CRYPTO 2003, 316-337.

Perković, T., ČAgalJ, M., Mastelić, T., SAXena, N., AND Begušić, D. 2011. Secure Initialization of Multiple Constrained Wireless Devices for an Unaided User. IEEE transactions on mobile computing.

Perrig, A., Szewczyk, R., Tygar, J., Wen, V., ANd Culler, D. 2002. Spins: Security protocols for sensor networks. Wireless networks 8, 5, 521-534.

Poon, C., ZhAnG, Y.-T., AND BAO, S.-D. 2006. A novel biometrics method to secure wireless body area sensor networks for telemedicine and m-health. IEEE Communications Magazine 44, 4, 73-81. 
PRASAD, R. AND SAXENA, N. 2008. Efficient device pairing using human-comparable synchronized audiovisual patterns. In Applied Cryptography and Network Security (ACNS). LNCS. 328-345.

Singh, K. AND MuthukKumarasamy, V. 2007. Authenticated key establishment protocols for a home health care system. In ISSNIP '07. 353-358.

StAJANO, F. AND ANDERSON, R. J. 2000. The resurrecting duckling: Security issues for ad-hoc wireless networks. In IWSP'00. 172-194.

TAN, C. C., WANG, H., Zhong, S., AND LI, Q. 2008. Body sensor network security: an identity-based cryptography approach. In ACM WiSec '08:. 148-153.

VAn Laerhoven, K., Schmidt, A., AND Gellersen, H.-W. 2002. Multi-sensor context aware clothing. In Wearable Computers, 2002. (ISWC 2002). 49-56.

VAUDENAY, S. 2005. Secure communications over insecure channels based on short authenticated strings. In Advances in Cryptology-CRYPTO 2005. Springer, 309-326.

Venkatasubramanian, K., BanerJee, A., And Gupta, S. 2010. Pska: Usable and secure key agreement scheme for body area networks. Information Technology in Biomedicine, IEEE Transactions on 14, 1, $60-68$.

Venkatasubramanian, K. AND Gupta, S. 2010. Physiological value-based efficient usable security solutions for body sensor networks. ACM Transactions on Sensor Networks (TOSN) 6, 4, 1-36.

Venkatasubramanian, K., Gupta, S., Jetley, R., AND Jones, P. 2010. Interoperable medical devices: Communication security issues. Pulse, IEEE 1, 2, 16-27.

Wong, C. K., Gouda, M., AND LAM, S. S. 1998. Secure group communications using key graphs. SIGCOMM Comput. Commun. Rev. 28, 4, 68-79.

ZhU, S., Setia, S., AND JAJOdia, S. 2003. Leap: efficient security mechanisms for large-scale distributed sensor networks. In CCS '03. 62-72.

ZHU, S., SETIA, S., AND JAJODIA, S. 2006. Leap+: Efficient security mechanisms for large-scale distributed sensor networks. ACM Transactions on Sensor Networks (TOSN) 2, 4, 500-528.

Zimmermann, P., Johnston, A., AND CALlas, J. March. 2006. Zrtp: Extensions to rtp for diffie-hellman key agreement for srtp draft-zimmermann-avt-zrtp-01. In Internet-draft. 